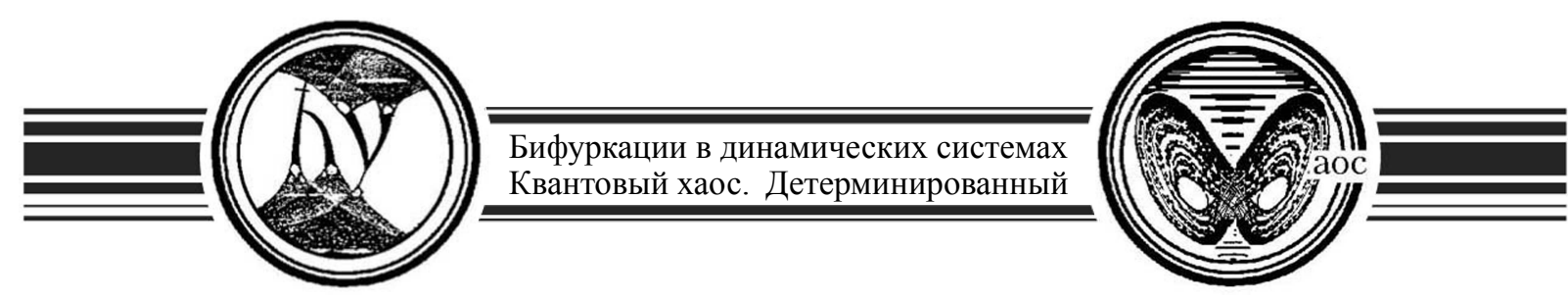

УДК 517.9:534.1

https://doi.org/10.18500/0869-6632-2019-27-6-39-62

\title{
Автогенератор грубого гиперболического хаоса
}

\author{
С.П. Кузнеиов \\ Институт радиотехники и электроники им. В.А. Котельникова РАН, Саратовский филиал \\ Россия, 410019 Саратов, Зеленая, 38 \\ E-mail: spkuz@yandex.ru \\ Поступила в редакцию 11.08.2019, принята к публикации 10.10.2019, опубликована 2.12.2019
}

Тема и цель исследования. Цель состоит в разработке автогенератора грубого хаоса, у которого на аттракторе реализуется динамика, близкая к потоку Аносова на многообразии отрицательной кривизны, в построении и анализе математической модели, а также проведении схемотехнического моделирования динамики с помощью программного продукта Multisim. Исследуемые модели. Сформулирована математическая модель, описываемая системой обыкновенных дифференциальных уравнений девятого порядка с алгебраической нелинейностью, и предложена схемотехническая реализация генератора хаоса. Результаты. Проведено численное исследование динамики, подтвердившее существование аттрактора, составленного из траекторий, близких к геодезическому потоку на поверхности отрицательной кривизны (Р-поверхность Шварца). Проведено схемотехническое моделирование электронного генератора. Представлены иллюстрации в виде осциллограмм, спектров мощности, изображений потока траекторий на аттракторе. Для математической модели проведено вычисление показателей Ляпунова и выполнена проверка гиперболической природы аттрактора с помощью анализа гистограмм углов пересечения устойчивых и неустойчивых многообразий. Обсуждение. Предложенный электронный генератор демонстрирует хаос, обладающий структурной устойчивостью в силу гиперболической природы аттрактора, что влечет нечувствительность динамики по отношению к малому изменению параметров системы, несовершенству изготовления, помехам. Этому гиперболическому аттрактору, кроме того, свойственна приблизительная равномерность растяжения и сжатия элементов фазового объема в непрерывном времени, что определяет хорошие спектральные свойства сигнала. Хотя рассмотрение проведено на уровне низкочастотного устройства, представляется возможной проработка и модификация схемы также для создания генераторов грубого хаоса высоких и сверхвысоких частот.

Ключевые слова: динамическая система, хаос, аттрактор, геодезический поток, динамика Аносова, гиперболичность, показатель Ляпунова.

Образеи цитирования: Кузнецов С.П. Автогенератор грубого гиперболического хаоса // Известия вузов. ПНД. 2019. T. 27, № 6. C. 39-62. https://doi.org/10.18500/0869-6632-2019-27-6-39-62

Финансовая поддержка. Работа выполнена при поддержке РНФ, грант № 17-12-01008. 


\title{
Self-oscillating system generating rough hyperbolic chaos
}

\author{
S. P. Kuznetsov \\ V.A. Kotelnikov Institute of Radio-Engineering and Electronics of RAS, Saratov Branch \\ 38 Zelenaya, Saratov 410019, Russia \\ E-mail: spkuz@yandex.ru \\ Received 11.08.2019, accepted for publication 10.10.2019, published 2.12.2019
}

\begin{abstract}
Topic and aim. The aim of the work is design of rough chaos generator, whose attractor implements dynamics close to Anosov flow on a manifold of negative curvature, as well as constructing and analyzing mathematical model, and conducting circuit simulation of the dynamics using the Multisim software. Investigated models. A mathematical model is considered that is a set of ordinary differential equations of the ninth order with algebraic nonlinearity, and a circuit representing the chaos generator is designed. Results. A numerical study of the dynamics of the mathematical model was carried out, which confirmed existence of the attractor composed of trajectories close to the geodesic flow on the surface of negative curvature (Schwarz P-surface). A circuit simulation of the electronic generator, in which the dynamics corresponds to the proposed mathematical model, is carried out. The illustrations of the system dynamics are presented in the form of oscilloscope traces, power spectra, pictures of the trajectory flow on the attractor. For the mathematical model, the Lyapunov exponents were calculated and the hyperbolic nature of the attractor was verified by analyzing histograms of the intersection angles of stable and unstable manifolds. Discussion. The proposed electronic generator demonstrates chaos with intrinsic structural stability due to hyperbolic nature of the attractor, which implies insensitivity of the dynamics with respect to small variations in the system parameters, manufacturing imperfections, and interferences. The hyperbolic attractor is characterized by approximate uniformity of stretching and compression for phase volume elements in continuous time, which determines rather good spectral properties of the signal. Although the consideration has been carried out for a low-frequency device, it seems possible to develop and modify the circuit also to create generators of rough chaos in high and ultra-high frequency bands.
\end{abstract}

Key words: dynamical system, chaos, attractor, geodesic flow, Anosov dynamics, hyperbolicity, Lyapunov exponent.

Reference: Kuznetsov S.P. Self-oscillating system generating rough hyperbolic chaos. Izvestiya VUZ. Applied Nonlinear Dynamics, 2019, vol. 27, no. 6, pp. 39-62. https://doi.org/10.18500/0869-6632-2019-27-6-39-62

Acknowledgements. The work was supported by Russian Science Foundation, grant no. 17-12-01008.

\section{Введение}

Считается, что динамический хаос обладает значительным потенциалом для приложений, таких как скрытая коммуникация [1-6], шифрование данных [7-10], генерация псевдослучайных чисел [11-14], создание радаров и сонаров [15-19].

Для практического применения существенно, чтобы хаотическая динамика была робастной [20-24], то есть не разрушалась при вариации параметров системы. Робастный хаос контрастирует с «хрупким хаосом» (fragile chaos) [25,26], который часто встречается в динамических системах и в математической теории находит выражение в концепции квазиаттрактора $[27,28]$. В последнем случае динамика, хотя и выглядит при численных расчетах или в эксперименте хаотической, но при более аккуратном рассмотрении характеризуется присутствием наряду с хаотическими орбитами регулярных притягивающих множеств («стоков») с узкими бассейнами притяжения, или же таковые появляются при сколь угодно малой вариации параметров.

К настоящему времени имеется обширная литература, посвященная проблеме генерации именно робастного хаоса. Для этого предлагалось использовать, например, системы, воспроизводящие динамику одномерных отображений с разрывной производной, кусочно-гладкие двумерные отображения, такие как отображения Лози [29,30] и Белых [31,32], а также динамику на аттракторах типа Лоренца (см. обзоры $[22,23]$.) Представляется, однако, что наиболее фундаментальный подход состоит в привлечении систем, демонстрирующих грубый гиперболический 
хаос, что дает возможность опираться на результаты глубокой математической теории, развитой во второй половине XX века [33-36].

Гиперболическая теория - это раздел теории динамических систем, доставляющий строгое обоснование возможности хаотического поведения для детерминированных систем как с дискретным временем (диффеоморфизмы), так и с непрерывным временем (потоки). Объектами исследования служат равномерно гиперболические инвариантные множества в фазовом пространстве, составленные исключительно из седловых траекторий, у которых векторы бесконечно малых возмущений представляются линейной комбинацией векторов сжимающего и растягивающего инвариантных подпространств касательного пространства. Сжимающее подпространство образовано векторами, нормы которых экспоненциально убывают при эволюции в прямом времени, а растягивающее - векторами, нормы которых экспоненциально убывают в обратном времени. Множества изображающих точек, приближающихся к данной траектории в прямом или обратном времени, отвечают, соответственно, устойчивому и неустойчивому многообразию этой траектории. Для гиперболического инвариантного множества эти многообразия могут пересекаться, но не должны иметь касаний.

Гиперболические инвариантные множества характеризуются свойством структурной устойчивости [37]. А именно, при малой вариации параметров или функций, фигурирующих в определении системы, динамика остается неизменной с точностью до непрерывной замены переменных. В случае физических или технических объектов это означает нечувствительность порождаемой динамики к малому изменению параметров системы, несовершенству изготовления, помехам и т.п.

Для консервативных систем гиперболический хаос представлен динамикой Аносова [34-36,38,39], когда равномерно гиперболическое инвариантное множество занимает компактное фазовое пространство полностью (для диффеоморфизма) или представляет собой поверхность постоянной энергии (для потока).

Для диссипативных систем гиперболическая теория вводит в рассмотрение притягивающие инвариантные множества с хаотической динамикой - равномерно гиперболические аттракторы [33-37, 40-50].

До недавнего времени примеры гиперболических хаотических аттракторов ограничивались математическими конструкциями. В последнее время предложены примеры систем, допускающих физическую реализацию [41-48], где гиперболический аттрактор получается для отображения, возникающего в рамках построения сечения Пуанкаре, тогда как для исходной потоковой системы аттрактором служит объект, именуемый надстройкой аттрактора этого отображения $[43,49]$. В этих системах в промежутках времени между прохождениями сечения Пуанкаре, как правило, не приходится говорить о хорошо выраженном равномерном по времени растяжении и сжатии для соответствующих подпространств векторов возмущения. Это негативно сказывается, в частности, на спектральных свойствах сигнала.

Представляет принципиальный интерес и, очевидно, существенное практическое значение для улучшения характеристик генераторов хаоса, построение систем с гиперболическими аттракторами, которые характеризовались бы равномерным (хотя бы приблизительно) растяжением и сжатием вдоль траекторий в непрерывном времени.

Чтобы подойти к решению этого вопроса, в качестве отправного пункта можно обратиться к потокам Аносова [38]. Простой формальный пример - задача о движении частицы в пространстве с отрицательной кривизной по геодезическим линиям метрики, заданной положительно определенной квадратичной формой с гладко зависящими от координат коэффициентами $[42,50]$. Такое движение характеризуется неустойчивостью по отношению к поперечным возмущениям, и если оно происходит в ограниченной области, оказывается хаотическим. Эта задача восходит к Адамару и была одним из главных источников вдохновения при создании гиперболической теории [51].

Кузнеиов С.П.

Известия вузов. ПНД, 2019, т. 27, № 6 
Заметим, что для приложений наиболее интересны автономные системы, выступающие как полноценные генераторы грубого хаоса, функционирование которых не требует присутствия переменных во времени воздействий.

Настоящая статья посвящена построению и анализу автономной системы, представляющей собой генератор гиперболического хаоса, характеризуемого приблизительно равномерным растяжением и сжатием вдоль траекторий в непрерывном времени. В разделе 1 , отправляясь от механической задачи с конфигурационным пространством в виде двумерного многообразия отрицательной кривизны, конструируется система дифференциальных уравнений, у которой на аттракторе реализуется динамика, близкая к геодезическому потоку Аносова. В разделе 2 конструируется и описывается электронная схема устройства, использующая операционные усилители, аналоговые умножители, полупроводниковые диоды, конденсаторы и резисторы. В разделе 3 представлены результаты схемотехнического моделирования динамики в программной среде Multisim [52] и проведено их сопоставление с результатами численного решения дифференциальных уравнений. Приводятся полученные для системы дифференциальных уравнений спектры показателей Ляпунова, а также поясняется методика и представлены результаты проверки гиперболической природы аттрактора с помощью анализа гистограмм углов пересечения устойчивых и неустойчивых многообразий.

\section{1. Математические модели с динамикой, близкой к потоку Аносова}

Обратимся сначала к системе, реализующей геодезический поток Аносова на двумерном многообразии отрицательной кривизны, пример которой доставляет тройной шарнирный механизм Тёрстона-Уикса-МакКея-Ханта $[53,54]$. При этом ограничимся упрощенной постановкой задачи, относящейся к определенному предельному случаю $[54,55]$.

Систему можно мыслить как составленную из трех ротаторов, мгновенные состояния которых характеризуются углами поворота $\left(\theta_{1}, \theta_{2}, \theta_{3}\right)$ и угловыми скоростями $\left(\dot{\theta}_{1}, \dot{\theta}_{2}, \dot{\theta}_{3}\right)$. Функция Лагранжа имеет вид

$$
L=\frac{1}{2}\left(\dot{\theta}_{1}^{2}+\dot{\theta}_{2}^{2}+\dot{\theta}_{3}^{2}\right)
$$

а условие механической голономной связи накладывается уравнением

$$
\cos \theta_{1}+\cos \theta_{2}+\cos \theta_{3}=0 .
$$

Последнее соотношение определяет в конфигурационном пространстве $\left(\theta_{1}, \theta_{2}, \theta_{3}\right)$ двумерную искривленную поверхность, известную как минимальная P-поверхность Швариа [56] (рис. 1). В силу периодичности по трем осям координат, переменные $\theta_{1,2,3}$ можно полагать определенными по модулю $2 \pi$ и трактовать движение как протекающее в компактной области кубической ячейке с длиной ребра $2 \pi$. Если отождествить противолежащие грани ячейки, получается компактное многообразие рода 3, классифицируемое в топологии как «сфера с тремя ручками» $[54,57]$.

Движения совершаются по геодезическим линиям на поверхности Шварца, гауссова кривизна которой дается выражением [54,55]

$$
K=-\frac{\cos ^{2} \theta_{1}+\cos ^{2} \theta_{2}+\cos ^{2} \theta_{3}}{2\left(\sin ^{2} \theta_{1}+\sin ^{2} \theta_{2}+\sin ^{2} \theta_{3}\right)^{2}} .
$$

За исключением восьми точек, где числитель обращается в нуль из-за одновременного равенства нулю всех трех косинусов $\left(\theta_{1}= \pm \pi / 2, \theta_{2}= \pm \pi / 2, \theta_{3}= \pm \pi / 2\right)$, кривизна $K$ всюду отрицательна, так что геодезический поток реализует динамику Аносова. 


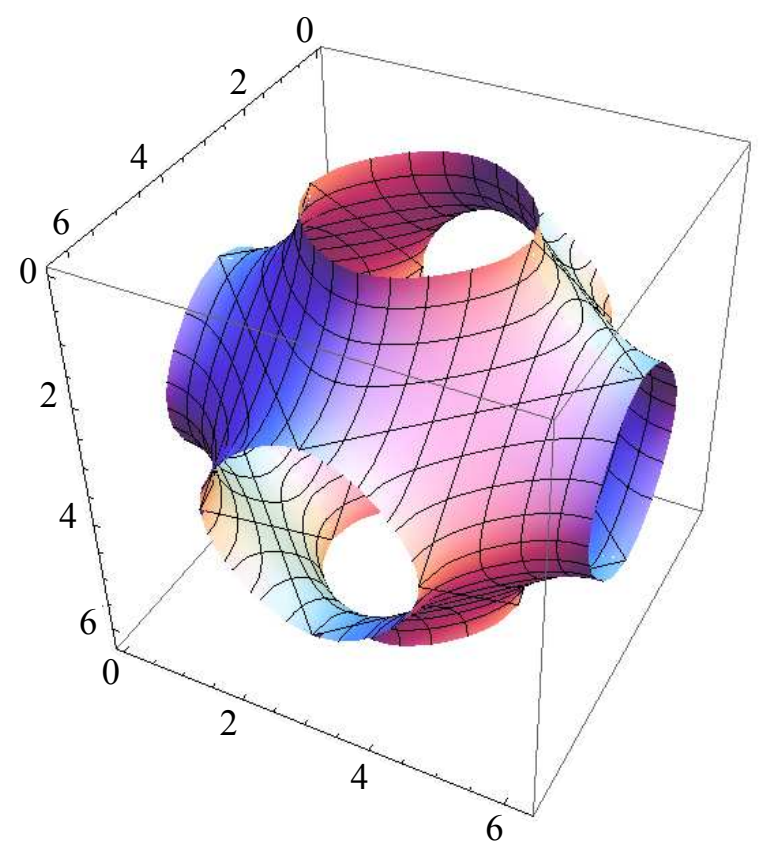

Рис. 1. Поверхность Шварца, изображенная в фундаментальной ячейке конфигурационного пространства $\left(\theta_{1}, \theta_{2}, \theta_{3}\right)$

Fig. 1. Schwarz surface depicted in the fundamental cell of the configuration space $\left(\theta_{1}, \theta_{2}, \theta_{3}\right)$

Используя стандартную для механических систем с голономными связями процедуру $[58,59]$, можно записать систему уравнений движения в виде

$$
\ddot{\theta}_{1}=\Lambda \sin \theta_{1}, \quad \ddot{\theta}_{2}=\Lambda \sin \theta_{2}, \quad \ddot{\theta}_{3}=\Lambda \sin \theta_{3},
$$

где множитель Лагранжа $\Lambda$ подлежит определению с учетом алгебраического условия механической связи. В нашем случае

$$
\Lambda=-\frac{\dot{\theta}_{1}^{2} \cos \theta_{1}+\dot{\theta}_{2}^{2} \cos \theta_{2}+\dot{\theta}_{3}^{2} \cos \theta_{3}}{\sin ^{2} \theta_{1}+\sin ^{2} \theta_{2}+\sin ^{2} \theta_{3}}
$$

Система формально шестимерная, но имеет два первых интеграла, один из которых дается уравнением (2), а другой - его производной по времени, так что размерность фазового пространства редуцируется до четырех. Кроме того, имеется интеграл энергии $W=(1 / 2)\left(\dot{\theta}_{1}^{2}+\dot{\theta}_{2}^{2}+\dot{\theta}_{3}^{2}\right)=$ $=$ const.

В работе [55] предложена автоколебательная система, динамика которой в установившемся режиме приблизительно соответствует геодезическому потоку на поверхности Шварца, хотя кинетическая энергия не остается постоянной, а совершает нерегулярные колебания около некоторого среднего уровня. Система построена из трех авторотаторов - элементов, состояние которых задается угловыми переменными $\theta_{1,2,3}$ и скоростями $z_{1,2,3}=\dot{\theta}_{1,2,3}$, причем для одного элемента в условиях изоляции установившееся движение отвечает вращению с постоянной скоростью в ту или иную сторону. Между авторотаторами включено взаимодействие посредством потенциала, имеющего минимум на поверхности Шварца: $U\left(\theta_{1}, \theta_{2}, \theta_{3}\right)=(1 / 2)\left(\cos \theta_{1}+\cos \theta_{2}+\cos \theta_{3}\right)^{2}$. Уравнения имеют вид

$$
\dot{\theta}_{i}=z_{i}, \quad \dot{z}_{i}=\mu z_{i}-v z_{i}^{3}+\left(\cos \theta_{1}+\cos \theta_{2}+\cos \theta_{3}\right) \sin \theta_{i}, \quad i=1,2,3,
$$

где $\mu$ и $v$ - параметры. Как было показано численно в работе [55], динамика в определенной области параметров остается гиперболической, хотя теперь следует говорить уже не о динамике 
Аносова консервативной системы, а об автоколебательном хаотическом режиме, отвечающем гиперболическому аттрактору.

При попытке реализовать на основе модели (6) электронное устройство главная проблема связана с тем, что нелинейность по переменным $\theta_{1,2,3}$ не алгебраическая, а выражается тригонометрическими функциями (синус и косинус).

В электронике такого рода нелинейности встречаются при рассмотрении контактов Джозефсона и систем типа цепочек фазовой автоподстройки [60-62].

Вопрос о том, можно ли построить модель с гиперболическим хаосом и как это сделать, используя контакты Джозефсона, остается открытым.

Электронное устройство на основе цепочек фазовой автоподстройки, которое в определенном приближении соответствует модели (6), описано в работах $[63,64]$. Наличие гиперболического аттрактора продемонстрировано в рамках численных расчетов в сочетании со схемотехническим моделированием в среде Multisim. Приходится отметить, что схема получается достаточно сложной (в частности, она включает блоки в виде генераторов частоты, управляемой напряжением). Кроме того, в силу самой природы цепочек фазовой автоподстройки система оказывается неавтономной: электронное устройство требует для своего функционирования опорного сигнала от источника переменного напряжения.

В настоящей работе развит альтернативный подход к построению электронного генератора, отправляясь от модели (6). Формально идея проста и состоит в том, чтобы воспользоваться заменой переменных, позволяющей исключить тригонометрические функции, и перейти к модели с алгебраической нелинейностью в виде системы уравнений с постоянными коэффициентами. Ценой является увеличение размерности фазового пространства системы, однако электронная схема получается проще, нежели описанная в работах $[63,64]$, и система оказывается автономной.

Если ввести новые переменные $x=\sin \theta, y=\cos \theta, z=\dot{\theta}$, то, очевидно,

$$
\dot{x}=y z, \quad \dot{y}=-x z,
$$

хотя при этом надо иметь в виду дополнительное условие $x^{2}+y^{2}=1$, выступающее как интеграл движения. Слегка модифицируя уравнения, запишем вместо (7)

$$
\dot{x}=\left(\kappa-\gamma x^{2}\right) x+y z, \quad \dot{y}=-x z,
$$

где к и $\gamma$ новые параметры. Их подбором можно обеспечить выполнение условия $x^{2}+y^{2} \approx 1$ (хотя это требование, как можно показать, не является непременно необходимым для правильного функционирования рассматриваемой ниже системы).

Введем новые переменные для всех трех подсистем, составляющих систему, и запишем вместо (6) следующие уравнения

$$
\begin{aligned}
& \dot{x}_{i}=\left(\kappa-\gamma x_{i}^{2}\right) x_{i}+y_{i} z_{i}, \\
& \dot{y}_{i}=-x_{i} z_{i}, \\
& \dot{z}_{i}=\mu z_{i}-v z_{i}^{3}+x_{i}\left(y_{1}+y_{2}+y_{3}\right), \quad i=1,2,3 .
\end{aligned}
$$

\footnotetext{
${ }^{1}$ Интересно отметить неожиданное сходство уравнений, описывающих каждую из трех подсистем, с трехмерной моделью, имеющей аттрактор типа Лоренца [64]: перекрестные члены в виде произведений двух переменных имеют такой же вид и располагаются на тех же местах. Отличие состоит в структуре линейных членов в уравнениях и в присутствии дополнительных кубических членов. Это наблюдение позволяет думать о возможности получить равномерно гиперболический хаос в системах, построенных как совокупность связанных элементов с аттракторами Лоренца. Также оно позволяет осознать, что по уровню сложности устройство в целом будет соответствовать системе трех связанных моделей Лоренца [15,65-67]. (Напомним, что сам по себе аттрактор Лоренца квалифицируется как псевдогиперболический или сингулярно гиперболический $[68,69]$ и, хотя и является робастным, не обладает в то же время полноценной структурной устойчивостью.)
} 
На рис. 2 иллюстрируется и сопоставляется вид траектории в пространстве угловых переменных $\left(\theta_{1}, \theta_{2}, \theta_{3}\right)$ для системы (4), (5) (панель $a$ ), системы (6) (панель $b$ ) и системы (9) (панель $c$ ). При построении графиков угловые переменные считались отнесенными к интервалу от 0 до $2 \pi$, то есть диаграмма отвечает фундаментальной ячейке, периодически повторяющейся при сдвиге по каждой из трех координатных осей на $2 \pi$.

На диаграмме ( $a$ ) траектория располагается в точности на поверхности Шварца (см. рис. 1) и соответствует геодезической. Из рисунка можно сделать качественное заключение об эргодической природе движения, присущей геодезическому потоку Аносова. Наличие хаоса подтверждается присутствием положительного показателя Ляпунова. Поскольку система (4), (5) не имеет внутреннего характерного временного масштаба, показатель Ляпунова пропорционален скорости, то есть $\lambda=k \sqrt{W}$, где $W=(1 / 2)\left(\dot{\theta}_{1}^{2}+\dot{\theta}_{2}^{2}+\dot{\theta}_{3}^{2}\right)$ - кинетическая энергия, а коэффициент $k$ определяется усредненной кривизной метрики и по результатам вычислений составляет для данной системы $k \approx 0.70$ [64].

Изображения на панелях $(b)$ и $(c)$ визуально выглядят практически так же, как и для потока Аносова на диаграмме $(a)$, что говорит о близости траекторий, реализующихся в установившемся режиме автоколебаний, к геодезическим линиям поверхности Шварца. При внимательном рассмотрении можно видеть, что изображения слегка «распушены» в поперечном направлении и не лежат в точности на поверхности Шварца. При выбранных здесь параметрах это, как оказывается, не ведет к нарушению гиперболической природы хаоса, что обусловлено, по существу, свойством грубости динамики Аносова, и будет подтверждено дальнейшим анализом.

Положительный показатель Ляпунова для установившихся режимов систем (6) и (9) по результатам численных расчетов, соответственно, $\lambda \approx 0.106$ и $\lambda \approx 0.107$. Это хорошо согласуется с показателем для геодезического потока Аносова $\lambda \approx 0.105$ при энергии, определенной как средняя полусумма квадратов угловых скоростей по результатам численного решении уравнений (6) и (9) $(1 / 2)\left(\dot{\theta}_{1}^{2}+\dot{\theta}_{2}^{2}+\dot{\theta}_{3}^{2}\right) \approx 0.0225$. (Более подробно спектры показателей Ляпунова обсуждаются в разделе 3.)
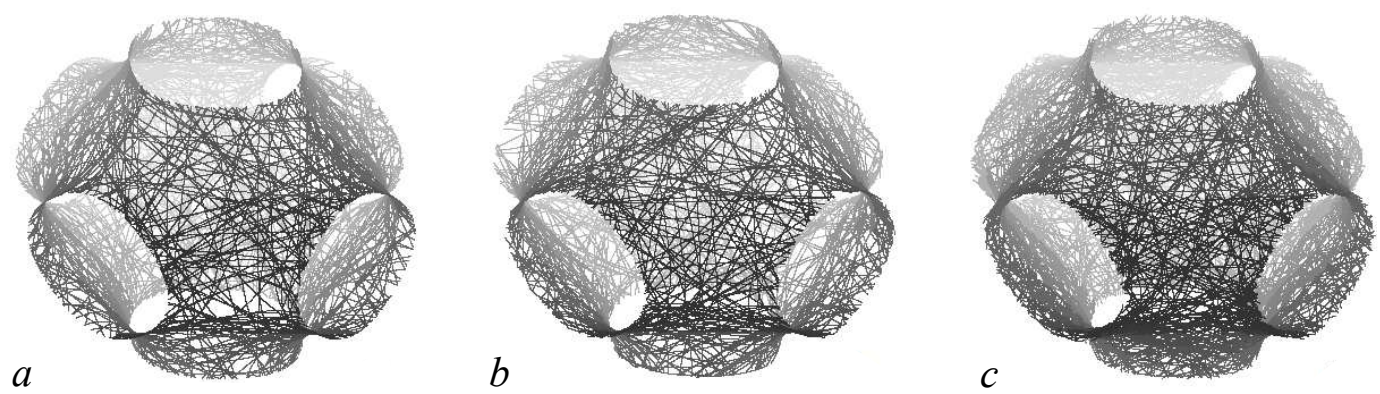

Рис. 2. Изображения траекторий, полученные при численном решении уравнений для консервативной системы (4), (5) при фиксированной энергии (a), для установившихся режимов хаотической динамики в шестимерной автоколебательной системе (6) при $\mu=0.04$ и $v=1.71(b)$ и в девятимерной системе (9) при $\mu=0.04, v=1.71, \kappa=0.024$ и $\gamma=0.03(c)$. Траектории представлены в фундаментальной ячейке в виде куба с ребром $2 \pi$ в трехмерном пространстве $\left(\theta_{1}, \theta_{2}, \theta_{3}\right)$. При пересечении одной из граней кубической ячейки траектория продолжается от противоположной грани, в силу периодичности по $\theta_{1,2,3}$. Для модели (9) угловые переменные вычислялись из соотношений $\theta_{i}=\arg \left(y_{i}+i x_{i}\right)$

Fig. 2. Pictures of trajectories obtained from numerical integration of the equations for the conservative system (4), (5) at a fixed energy (a), for sustained chaotic dynamics in the six-dimensional self-oscillating system (6) at $\mu=0.04$ and $v=1.71$ (b) and in the nine-dimensional system (9) with $\mu=0.04, \nu=1.71, \kappa=0.024$ и $\gamma=0.03(c)$. The trajectories are depicted in the fundamental cell that is a cube with an edge $2 \pi$ in the three-dimensional space $\left(\theta_{1}, \theta_{2}, \theta_{3}\right)$. When crossing one of the faces of the cubic cell, a trajectory continues from the opposite face, due to the periodicity in $\theta_{1,2,3}$. For the model (9), the angular variables were calculated from $\theta_{i}=\arg \left(y_{i}+i x_{i}\right)$

Кузнецов С.П.

Известия вузов. ПНД, 2019, т. 27, № 6 


\section{2. Электронный генератор хаоса}

На рис. 3 показана схема автогенератора, описание динамики которого приводится к уравнениям (9). Схема содержит три одинаковых блока D1, D2, D3. Выходными сигналами на терминалах каждого блока служат напряжения, отвечающие переменным $x_{i}, y_{i}, z_{i}, i=1,2,3$, а на входной терминал подается сигнал $Y=-\left(y_{1}+y_{2}+y_{3}\right)$ с выхода инвертирующего сумматора на операционном усилителе ОА.

Для вывода уравнений обратимся к схеме индивидуального блока, показанной на рис. 3 внизу. Имея в виду, что мгновенные значения напряжений на конденсаторах $\mathrm{C} 1, \mathrm{C} 2$ и С 3 состав-

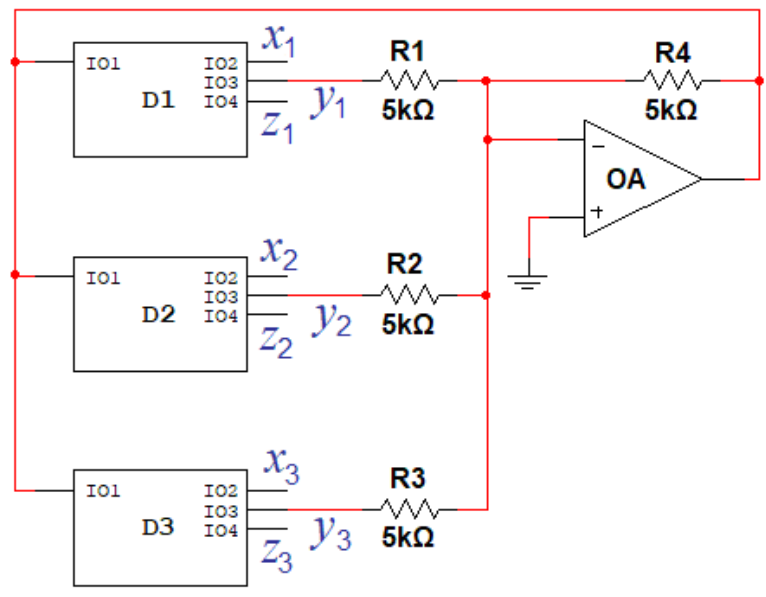

$a$

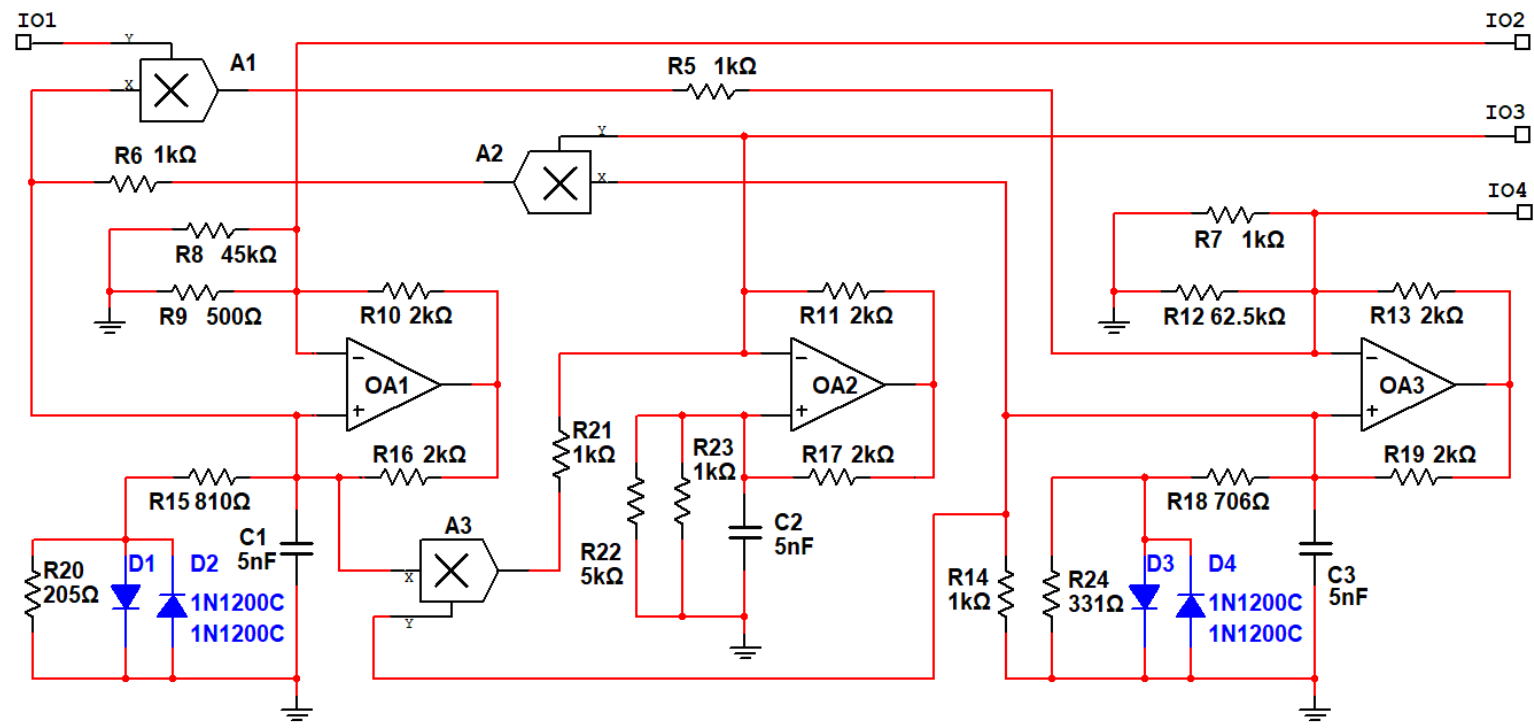

$b$

Рис. 3. Схема генератора хаоса, описываемого уравнениями (9) (a), и схема блока D (b)

Fig. 3. Circuit diagram for chaos generator governed by equations (9) $(a)$ and diagram for block D $(b)$ 
ляют $x, y$ и $z$, запишем условия нулевого суммарного тока на инвертирующем и неинвертирующем входе для операционного усилителя OA1

$$
\begin{aligned}
\left(R_{8}^{-1}+R_{9}^{-1}\right) x & =\left(U_{1}-x\right) R_{10}^{-1}, \\
C_{1} \dot{x}+a x+b x^{3} & =\left(\frac{1}{10} y z-x\right) R_{6}^{-1}+\left(U_{1}-x\right) R_{16}^{-1},
\end{aligned}
$$

для усилителя ОА2

$$
\begin{aligned}
& \left(y-\frac{1}{10} x z\right) R_{21}^{-1}+y R_{0}^{-1}=\left(U_{2}-y\right) R_{11}^{-1} \\
& C_{2} \dot{y}+R_{22}^{-1} y+R_{23}^{-1} y=\left(U_{2}-y\right) R_{17}^{-1}
\end{aligned}
$$

и для усилителя ОА3

$$
\begin{aligned}
& \left(R_{7}^{-1}+R_{12}^{-1}\right) z=\left(\frac{1}{10} x Y-z\right) R_{5}^{-1}+\left(U_{3}-z\right) R_{13}^{-1} \\
& C_{3} \dot{z}+z R_{14}^{-1}+\bar{a} z+\bar{b} z^{3}=\left(U_{3}-z\right) R_{19}^{-1}
\end{aligned}
$$

Здесь $U_{1,2,3}$ - напряжения на выходе операционных усилителей; $Y$ - напряжение на входном терминале IO1; $a, b, \bar{a}, \bar{b}$ - коэффициенты, задающие вольт-амперные характеристики нелинейных элементов на диодах D1, D2 и D3, D4 (см. Приложение). Коэффициент передачи аналоговых умножителей A1, A2, A3 задан равным 1/10, как это обычно принято. В первом уравнении (10) присутствует член, отвечающий за утечку через терминал IO3 тока, который выражается соотношением $y R_{0}^{-1}$, где $R_{0}$ - единое обозначение для равных сопротивлений $R_{1,2,3}$ в общей схеме на рис. 3 вверху.

Имея в виду равенство емкостей $C_{1,2,3}=5 \mathrm{nF}$, вводим для них общее обозначение $C$. Аналогично для одинаковых сопротивлений $R_{5,6,7,14,21,23}=1 \mathrm{k} \Omega$ используем обозначение $R$, а для вдвое меньшего сопротивления $R_{9}$, соответственно, $R / 2$. Кроме того, полагаем, что сопротивления $R_{15,20}$ и $R_{18,24}$, определяющие характеристики нелинейных элементов, подобраны так, чтобы обеспечить $a=\bar{a}=R^{-1}$. Учитывая эти замечания, а также равенство сопротивлений $R_{10,16}, R_{11,17}, R_{13,19}$ и сопротивлений $R_{0}$ и $R_{22}$, представим уравнения (10)-(12) как

$$
\begin{aligned}
& 10 R C \dot{x}=10 R R_{8}^{-1} x-10 R b x^{3}+y z, \\
& 10 R C \dot{y}=-x z, \\
& 10 R C \dot{z}=10 R R_{12}^{-1} z-10 R \bar{b} z^{3}-x Y .
\end{aligned}
$$

Запишем теперь три комплекта уравнений вида (13) для трех блоков, составляющих полную схему, снабдив переменные соответствующими индексами, подставим $Y=-\left(y_{1}+y_{2}+y_{3}\right)$ и введем параметры

$$
\kappa=10 R R_{8}^{-1}, \quad \gamma=10 R b, \quad \mu=10 R R_{12}^{-1}, \quad \nu=10 R \bar{b}
$$

В результате получаем в точности уравнения (9), в которых за единицу времени следует принять величину $10 R C=50 \mu \mathrm{s}$, а переменные $x_{1,2,3}, y_{1,2,3}, z_{1,2,3}$ представляют собой непосредственно напряжения в вольтах на выходных терминалах блоков D1, D2, D3. 


\section{3. Схемотехническое моделирование и численные результаты}

На рис. 4 показаны реализации напряжений на выходных клеммах одного из блоков D, скопированные с экрана виртуального осциллографа при моделировании динамики схемы в среде Multisim. ${ }^{2}$ Визуально они выглядят как хаотические, без видимого повторения форм.

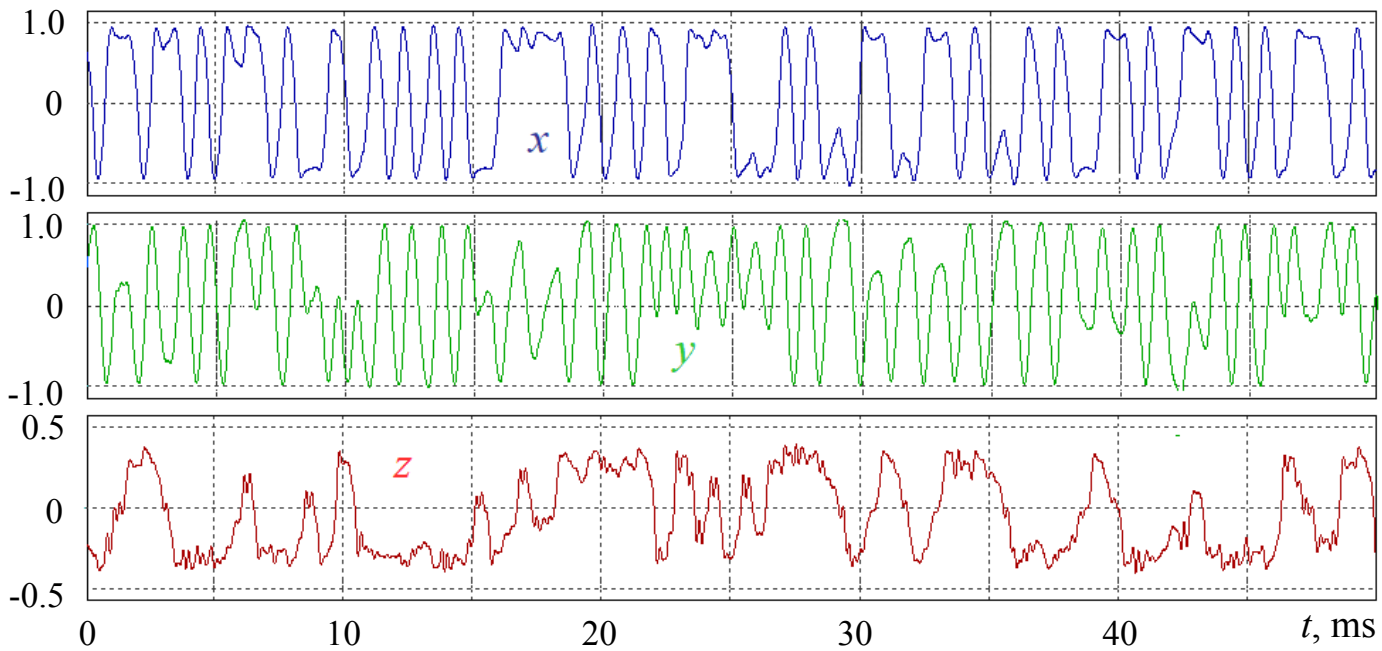

Рис. 4. Реализации напряжений на выходных терминалах блока D1 в установившемся режиме, полученные с виртуального осциллографа при моделировании в среде Multisim. По вертикальной оси отложены напряжения в вольтах. Номиналы компонентов схемы отвечают рис. 3

Fig. 4. Waveforms of voltages at the output terminals of the D1 unit in the sustained regime obtained from the screen of a virtual oscilloscope during modeling in the Multisim environment. Along the vertical axes, the voltages are plotted in volts. The values of the components of the circuit correspond to Fig. 3
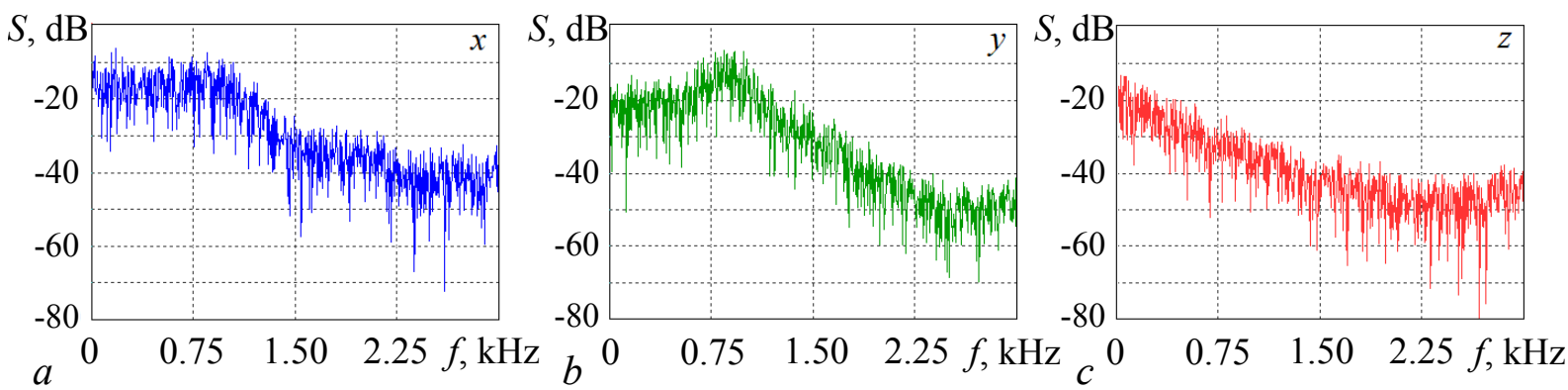

Рис. 5. Спектры мощности для напряжения на выходных терминалах блока D1, полученные как снимок с экрана виртуального анализатора спектра при моделировании в среде Multisim. Опорный уровень при определении спектральной плотности в децибелах выбран произвольно, но одинаковым образом для всех трех сигналов

Fig. 5. The power spectra for the voltages at the output terminals of the D1 block obtained as screenshots from the virtual spectrum analyzer during modeling in the Multisim environment. The reference level of the spectral density in dB is chosen arbitrarily, but in the same way for all three signals

На рис. 5 показаны спектры сигналов $x, y, z$, полученные с помощью виртуального анализатора спектра. Видно, что спектр сплошной, как это и должно быть для хаотического процесса.

\footnotetext{
${ }^{2}$ При моделировании в среде Multisim имеется проблема запуска системы из-за большого времени ухода от тривиального состояния равновесия. Один из вариантов состоит в том, чтобы использовать три версии блока $\mathrm{D}$, схемы которых идентичны, но различаются начальные напряжения на присутствующих в схеме конденсаторах, и включить опцию «User defined initial conditions».
} 
Он характеризуется медленным спаданием спектральной плотности с ростом частоты и хорошим качеством в смысле отсутствия локальных пиков и провалов. В силу симметрии схемы, вид реализаций и спектров выходных сигналов для всех трех блоков D1, D2, D3 аналогичен.

Можно проверить, что динамика электронного устройства аналогична динамике в исходной системе (4), (5) в том смысле, что траектория в пространстве координатных переменных $\left(\theta_{1}, \theta_{2}, \theta_{3}\right)$ располагается вблизи поверхности Шварца. На рис. 6 показаны диаграммы, полученные обработкой результатов схемотехнического моделирования в среде Multisim. Для их построения сигналы с выхода трех блоков D подаются на входы виртуальных осциллографов, и в процессе функционирования схемы производится запись этих сигналов в файл для последующей обработки. Для построения диаграмм по записанным данным в каждый момент времени вычислялись три угловые переменные $\theta_{k}=\arg \left(y_{k}+i x_{k}\right), k=1,2,3$, и соответствующая точка отображалась на графике.

Представленные диаграммы относятся к двум режимам электронного генератора. В одном из них степень близости к геодезическому потоку выше - изображение «распушено» в поперечном направлении слабо $(a)$, а во втором случае - сильнее $(b)$. Второй режим соответствует номиналам компонентов, указанных на рис. 3 , а первый - схеме с измененными номиналами нескольких резисторов $\left(R_{8}=417 \mathrm{k} \Omega, R_{12}=250 \mathrm{k} \Omega, R_{15}=893 \mathrm{k} \Omega, R_{20}=112 \Omega\right)$. В обеих ситуациях аттрактор гиперболический, что подтверждается анализом на основе критерия углов в рамках расчетов на основе численного решения дифференциальных уравнений.

В рамках схемотехнического моделирования затруднительно исследовать некоторые характеристики, например, показатели Ляпунова, и не представляется возможным провести расчеты по проверке гиперболической природы хаоса. Поэтому обратимся к сопоставлению полученных результатов с динамикой модели (9), для которой соответствующий анализ в численных расчетах можно выполнить.

Используя номиналы компонентов схемы на рис. 3 и формулы предыдущего раздела, находим параметры, фигурирующие в уравнениях (9): $\mu=0.16, v=1.71, \kappa=0.22, \gamma=0.3$.

На рис. 7 показаны графики зависимости переменных $x_{1}, y_{1}, z_{1}$ от безразмерного времени, построенные по результатам численного решения уравнений (9).

Хотя точного совпадения ожидать не приходится из-за хаотической природы динамики и ее чувствительности к малым вариациям начальных условий, полученные при численном решении уравнений графики находятся в разумном соответствии с осциллограммами на рис. 4 , полученными при моделировании в среде Multisim (общий вид реализаций, характерные масштабы по осям абсцисс и ординат).
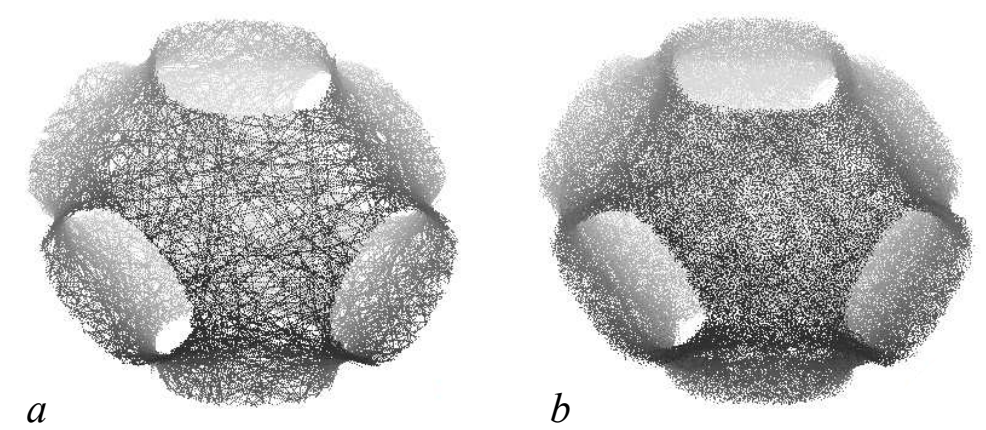

Рис. 6. Вид траекторий в трехмерном пространстве $\left(\theta_{1}, \theta_{2}, \theta_{3}\right)$ по результатам моделирования в среде Multisim. Методика построения диаграмм описана в тексте

Fig. 6. Pictures of trajectories in three-dimensional space $\left(\theta_{1}, \theta_{2}, \theta_{3}\right)$ as obtained by processing data of modeling in the Multisim environment with the technique described in the text 


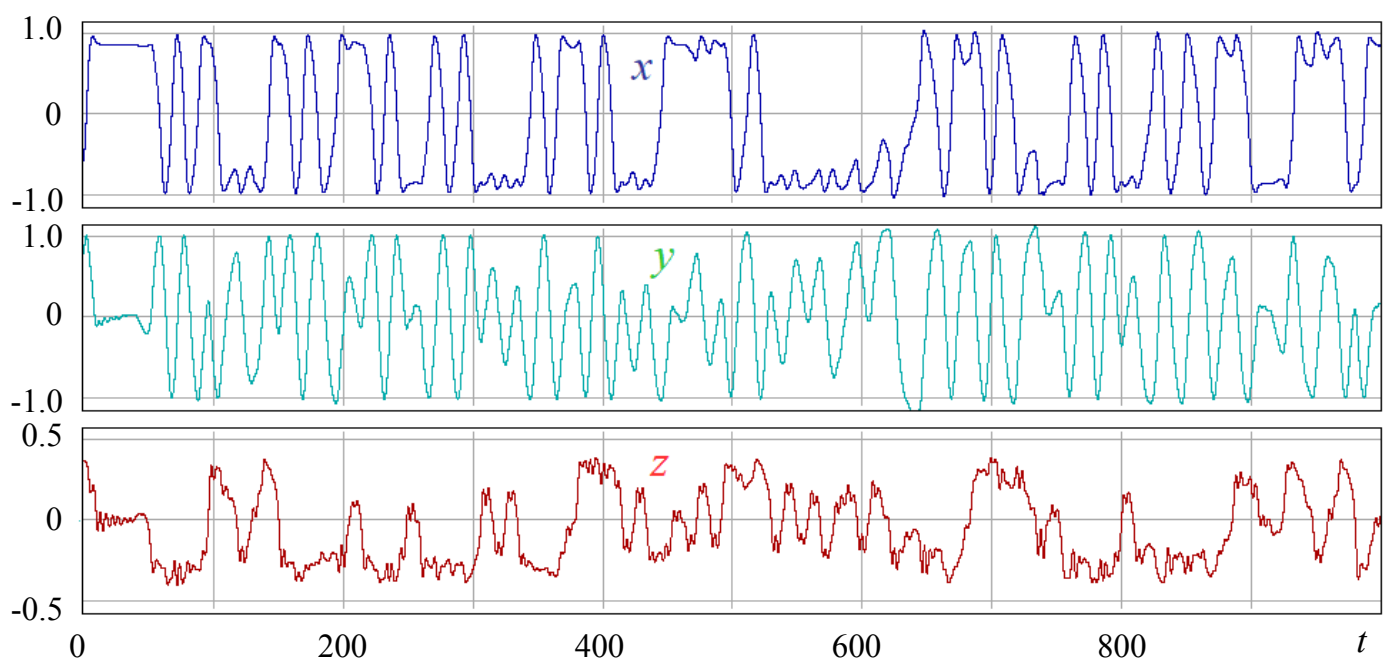

Рис. 7. Временные зависимости переменных $x_{1}, y_{1}, z_{1}$, полученные при численном решении уравнений (9). Параметры: $\mu=0.16, v=1.71, \kappa=0.22, \gamma=0.3$. Интервал безразмерного времени $t=1000$ соответствует $50 \mathrm{~ms}$, как следует из указанного в разделе 2 правила пересчета

Fig. 7. Time dependences of the variables $x_{1}, y_{1}, z_{1}$, obtained numerically from integration of the equations (9). Parameters: $\mu=0.16, v=1.71, \kappa=0.22, \gamma=0.3$. The dimensionless time interval $t=1000$ corresponds to $50 \mathrm{~ms}$, as follows from the rescaling rule specified in Section 2

На рис. 8 показаны спектры сигналов, полученные обработкой данных численного решения уравнений (9) согласно методике, принятой в теории статистического анализа характеристик случайных процессов [71,72]. Распределения спектральной плотности, как можно видеть, хорошо согласуются с видом спектров при моделировании в среде Multisim (см. рис. 5).

На рис. 9 показан график зависимости от параметра $\mu$ всех девяти показателей Ляпунова при фиксированных $v=1.71, \kappa=0.22, \gamma=0.3$ согласно численным расчетам для модели (9) с помощью традиционного алгоритма [73-75]. В широком интервале по параметру имеется один положительный, два близких к нулю, и остальные отрицательные показатели Ляпунова, то есть реализуется хаос. При $\mu \approx 0.58$ хаос исчезает и возникает режим, у которого старший показатель
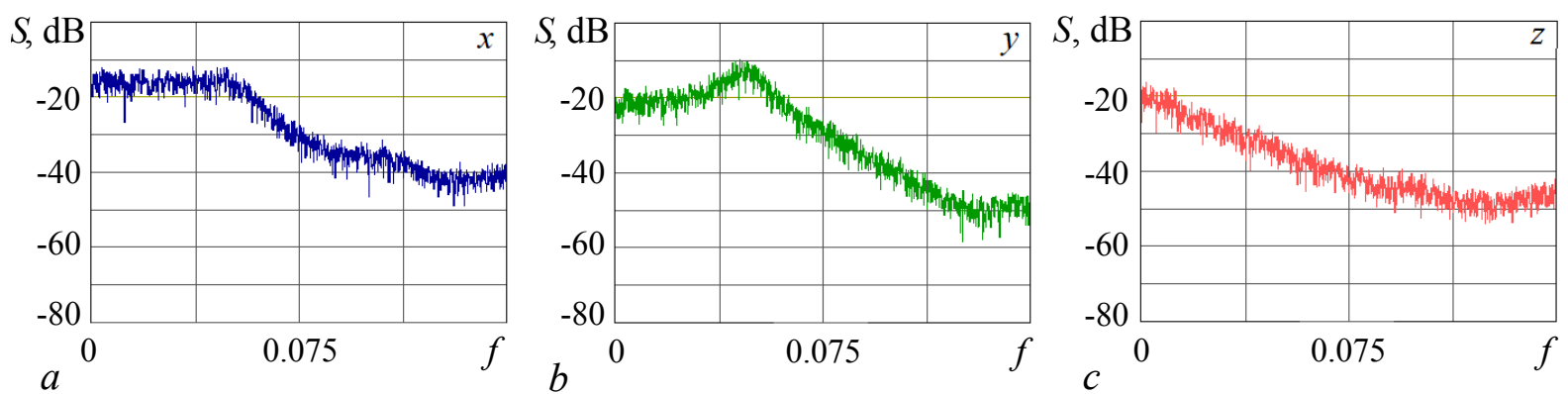

Рис. 8. Спектры мощности сигналов, отвечающих переменным $x_{1}, y_{1}, z_{1}$, полученные обработкой данных численного интегрирования системы уравнений (9). Параметры: $\mu=0.16, v=1.71, \kappa=0.22, \gamma=0.3$. Интервал безразмерной частоты $f=0.15$ отвечает $3 \mathrm{kHz}$ в соответствии с указанным в разделе 2 правилом пересчета от размерного времени к безразмерному. Опорный уровень при определении спектральной плотности в децибелах выбран произвольно, но одинаковым образом для всех трех сигналов

Fig. 8. Power spectra of the signals corresponding to the variables $x_{1}, y_{1}, z_{1}$ obtained by processing the data of numerical integration of the equations (9) at parameters $\mu=0.16, v=1.71, \kappa=0.22, \gamma=0.3$. Interval of the dimensionless frequency $f=0.15$ corresponds to $3 \mathrm{kHz}$ in accordance with the converting rule from time to dimensionless time specified in Section 2 . The reference level in $\mathrm{dB}$ is chosen arbitrarily, but in the same way for all three signals 
Ляпунова нулевой, что соответствует периодическому движению на предельном цикле. Расчеты показывают, что гиперболичность хаоса имеет место в левой части представленного интервала и нарушается, примерно начиная с $\mu \approx 0.34$, см. ниже. Отметим, что в этой области зависимость показателей от параметра плавная, без изрезанности.

Идея проверки гиперболичности инвариантного множества динамической системы на основе статистики углов между устойчивыми и неустойчивыми подпространствами на фазовой траектории была предложена в работе [39] и впоследствии использовалась применительно к аттракторам, например, в работах [43,44,55,77-81]. Методика состоит в том, что в точках одной и той же принадлежащей инвариантному множеству типичной траектории определяются углы между подпространствами векторов малых возмущений при про-

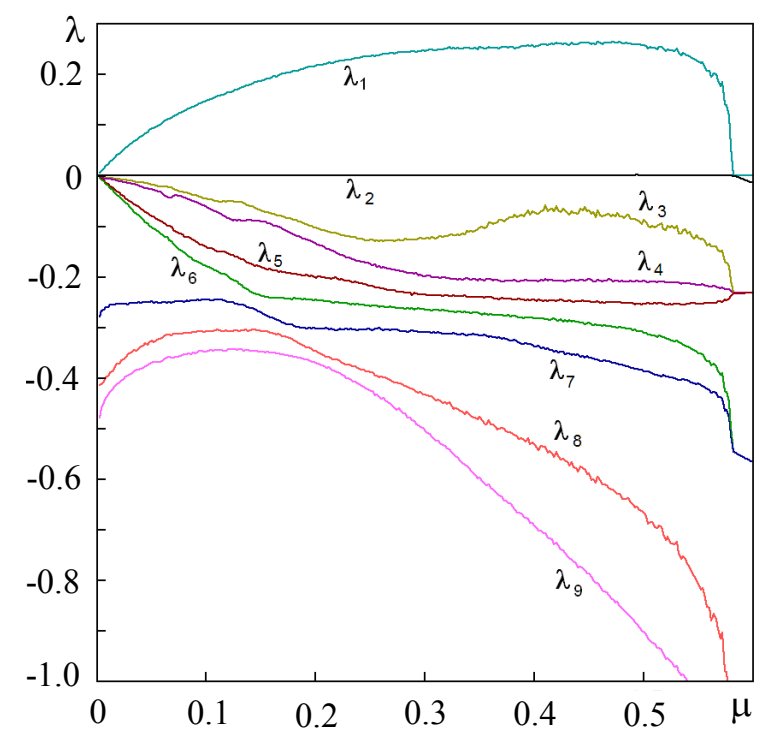

Рис. 9. Показатели Ляпунова системы (9) в зависимости от $\mu$ при $v=1.71, \kappa=0.22, \gamma=0.3$

Fig. 9. Lyapunov exponents of system (9) depending on $\mu$ for $v=1.71, \kappa=0.22, \gamma=0.3$ ходе вперед и назад во времени, и анализируется их распределение. Если нет углов близких к нулю, то тем самым подтверждается гиперболичность инвариантного множества. Если же обнаруживается положительная вероятность нулевых углов, то касания между устойчивыми и неустойчивыми многообразиями присутствуют, и гиперболичности нет.

Процедура начинается с вычисления опорной орбиты на аттракторе за достаточно большой временной интервал, для чего проводится численное решение уравнений, которые здесь уместно записать кратко как $\dot{\mathbf{x}}=\mathbf{F}(\mathbf{x}, t)$, где $\mathbf{x}-$ девятимерный вектор состояния системы.

Интересуясь одномерным подпространством, ассоциирующимся с наибольшим показателем Ляпунова, интегрируем линеаризованное уравнение для вектора возмущения $\dot{\tilde{\mathbf{x}}}=\mathbf{F}^{\prime}(\mathbf{x}(t), t) \tilde{\mathbf{x}}$ вдоль найденной траектории $\mathbf{x}(t)$ и, нормируя векторы $\tilde{\mathbf{x}}$ на единицу на каждом шаге численного интегрирования $n$, получаем набор векторов $\left\{\mathbf{x}_{n}\right\}$. Далее, проводим интегрирование линейного уравнения $\dot{\mathbf{u}}=-\left[\mathbf{F}^{\prime}(\mathbf{x}(t), t)\right]^{\mathrm{T}} \mathbf{u}$, где Т означает сопряжение, в обратном времени вдоль той же самой опорной траектории [78]. Это позволяет получить набор нормированных на единицу векторов $\left\{\mathbf{u}_{n}\right\}$, определяющих ортогональное дополнение к сумме устойчивого и нейтрального подпространств векторов возмущения на опорной траектории.

Теперь для оценки угла ф между подпространствами на каждом $n$-ом шаге вычисляем угол $\beta_{n} \in[0, \pi / 2]$ между векторами $\tilde{\mathbf{x}}_{n}, \mathbf{u}_{n}$ и полагаем $\phi_{n}=\pi / 2-\beta_{n}$.

На рис. 10 показаны полученные численно гистограммы распределения углов, для аттракторов системы (9) при значениях безразмерных параметров, отвечающих двум вариантам выбора номиналов схемы, для которых построены изображения на рис. 6. Диаграммы на рис. 6 можно сравнить с показанными на вставках изображениями траекторий в пространстве угловых переменных $\left(\theta_{1}, \theta_{2}, \theta_{3}\right)$, построенными по результатам численного решения уравнений (9).

Для сравнения на рис. 11 представлены гистограммы, полученные в ситуации, когда гиперболичность нарушается, что имеет место при достаточно больших величинах параметра $\mu$. В самом деле, эти гистограммы демонстрируют присутствие углов вблизи нуля, что говорит о наличии касаний устойчивых и неустойчивых многообразий и негиперболической природе аттрактора. Поскольку в исходной системе - геодезическом потоке на поверхности отрицательной 

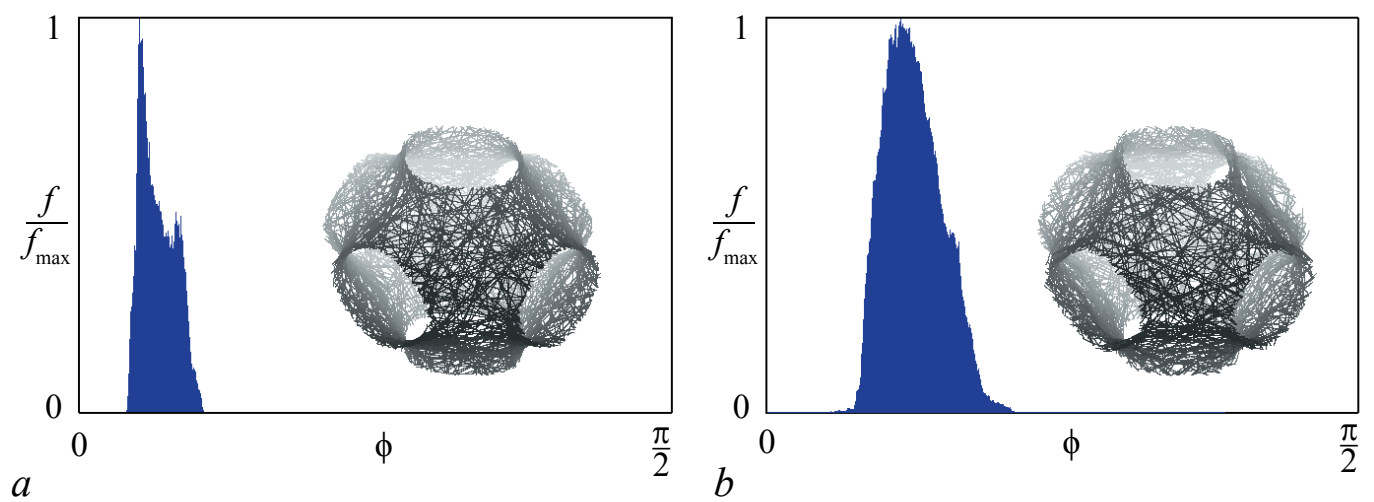

Рис. 10. Полученные численно гистограммы распределения углов между устойчивыми и неустойчивыми подпространствами в модели (9) при $\mu=0.04, v=1.71, \kappa=0.024, \gamma=0.03$ (a) и при $\mu=0.16, v=1.71, \kappa=0.22$, $\gamma=0.3(b)$. Распределения не включают нулевых углов $\phi$, то есть гиперболичность подтверждается. На вставках показан вид траектории в пространстве угловых переменных $\left(\theta_{1}, \theta_{2}, \theta_{3}\right)$, построенные по результатам численного решения уравнений (9)

Fig. 10. Numerically obtained histograms for distributions of angles between stable and unstable subspaces in model (9) for $\mu=0.04, v=1.71, \kappa=0.024, \gamma=0.03(a)$ and for $\mu=0.16, v=1.71, \kappa=0.22, \gamma=0.3(b)$. The distributions exclude zero angles $\phi$, i.e., the hyperbolicity is confirmed. The insets show pictures of trajectory in the space of angular variables $\left(\theta_{1}, \theta_{2}, \theta_{3}\right)$, plotted according to the data of numerical integration of the equations ( 9 )
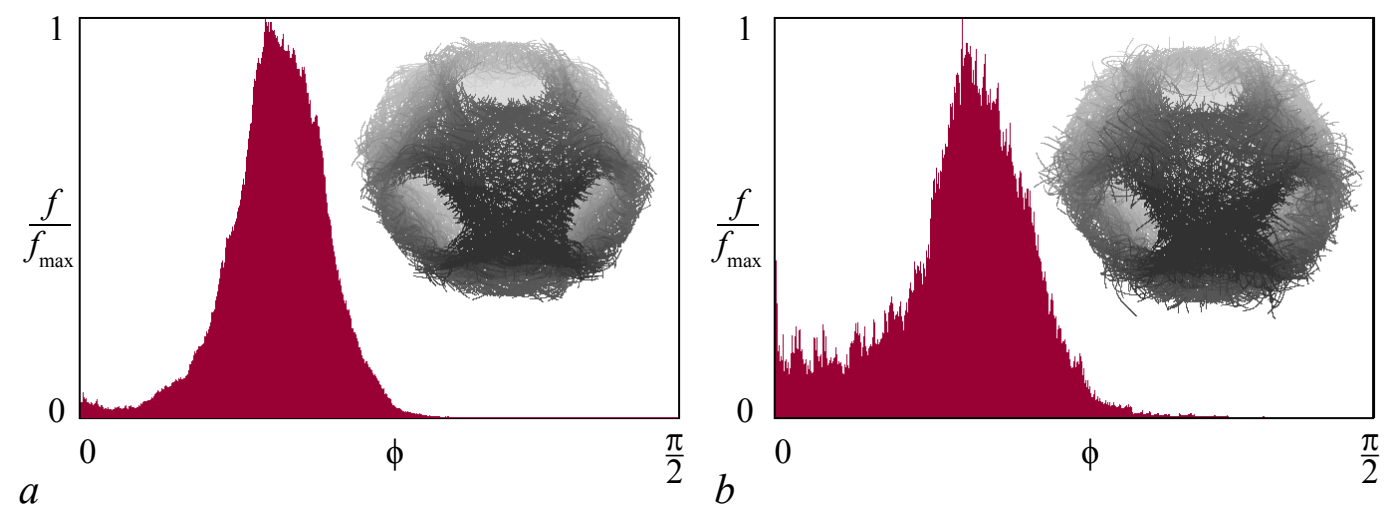

Рис. 11. Полученные численно гистограммы распределения углов между устойчивыми и неустойчивыми подпространствами в модели (9) при $v=1.71, \kappa=0.22, \gamma=0.3, \mu=0.34(a)$ и $\mu=0.48(b)$. Распределения включают нулевые углы $\phi$, - гиперболичности нет. На вставках показан вид траектории в пространстве угловых переменных $\left(\theta_{1}, \theta_{2}, \theta_{3}\right)$, построенные по результатам численного решения уравнений (9)

Fig. 11. Numerically obtained histograms for distributions of angles between stable and unstable subspaces in model (9) for $v=1.71, \kappa=0.22, \gamma=0.3, \mu=0.34(a)$ and $\mu=0.48(b)$. Distributions include zero angles $\phi$ indicating absence of the hyperbolicity. The insets show pictures of trajectory in the space of angular variables $\left(\theta_{1}, \theta_{2}, \theta_{3}\right)$ plotted according to the data of numerical integration of the equations (9) 
кривизны этого не наблюдается, естественно полагать, что отсутствие гиперболичности обусловлено возникновением существенных отклонений принадлежащих аттрактору траекторий от поверхности Шварца, заданной уравнением $\cos \theta_{1}+\cos \theta_{2}+\cos \theta_{3}=0$. Это действительно видно на вставках, показывающих сильно «распушенные» изображения траекторий в пространстве угловых переменных.

\section{Заключение}

В настоящей работе предложен подход к построению электронного генератора грубого хаоса, отправляясь от задачи о геодезическом потоке на поверхности отрицательной кривизны, где реализуется гиперболическая динамика Аносова. Рассмотрена схема генератора хаоса и проведено моделирование ее функционирования в среде Multisim. Также представлены модельные уравнения, описывающие систему, и выполнено компьютерное исследование порождаемой ими хаотической динамики.

В отличие от систем, в которых гиперболический аттрактор реализовался для отображения Пуанкаре [41-48], в данном случае гиперболичность обусловлена спецификой динамики в непрерывном времени. В отличие от рассмотренной ранее схемы, порождающей такой же тип гиперболической динамики $[63,64]$, предложенное здесь устройство проще по конструкции и представляет собой автономную систему, не требующую для своего функционирования внешнего периодического воздействия.

Таким образом, в настоящей работе впервые показано, как можно реализовать электронный автогенератор грубого хаоса, где на гиперболическом аттракторе имеет место приблизительная равномерность растяжения и сжатия элементов фазового объема в непрерывном времени, что определяет хорошие спектральные свойства сигнала.

Хотя описанная здесь конкретная система функционирует в низкочастотном диапазоне (килогерцы), представляется возможной проработка и модификация схемы также для создания генераторов грубого хаоса высоких и сверхвысоких частот.

Приложение

\section{Вольт-амперная характеристика составного элемента из резисторов и диодов}

Рассмотрим нелинейный элемент, реализованный с использованием пары параллельно включенных полупроводниковых диодов и резисторов по схеме, показанной на рис. А1, $a$.

Пусть на элемент подается напряжение $U$. Ток через резистор $R_{0}$ обозначим $I$, а напряжение на диодах $u$. Примем, что ток через диодную пару дается выражением $I_{D 12}=r_{D}^{-1} u\left(1+\beta u^{2}\right)$. Для диода $1 \mathrm{~N} 1200 \mathrm{C}$, согласно результатам моделирования в среде Multisim, аппроксимация получается удовлетворительной при выборе коэффициентов $r_{D} \approx 2.6 \mathrm{k} \Omega$ и $\beta=60 \mathrm{~V}^{-2}$. Для полного тока можно записать

$$
I=(U-u) R_{0}^{-1}=u r_{0}^{-1}+u r_{D}^{-1}\left(1+\beta u^{2}\right) .
$$

Получим выражение для $I$ через $U$ в виде разложения в ряд, содержащего члены первой и третьей степени. Для этого сделаем подстановку $I=a U+b U^{3}, u=c U+d U^{3}$ и выпишем вытекающие из формулы (A1) соотношения

$$
\begin{aligned}
& a U+b U^{3}=U R_{0}^{-1}-c R_{0}^{-1} U-d R_{0}^{-1} U^{3}, \\
& a U+b U^{3}=\left(c U+d U^{3}\right)\left(r_{0}^{-1}+r_{D}^{-1}\right)+\beta r_{D}^{-1} c^{3} U^{3} .
\end{aligned}
$$

Кузнецуов С.П. 


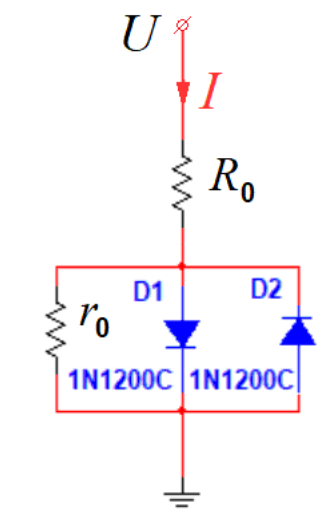

$a$

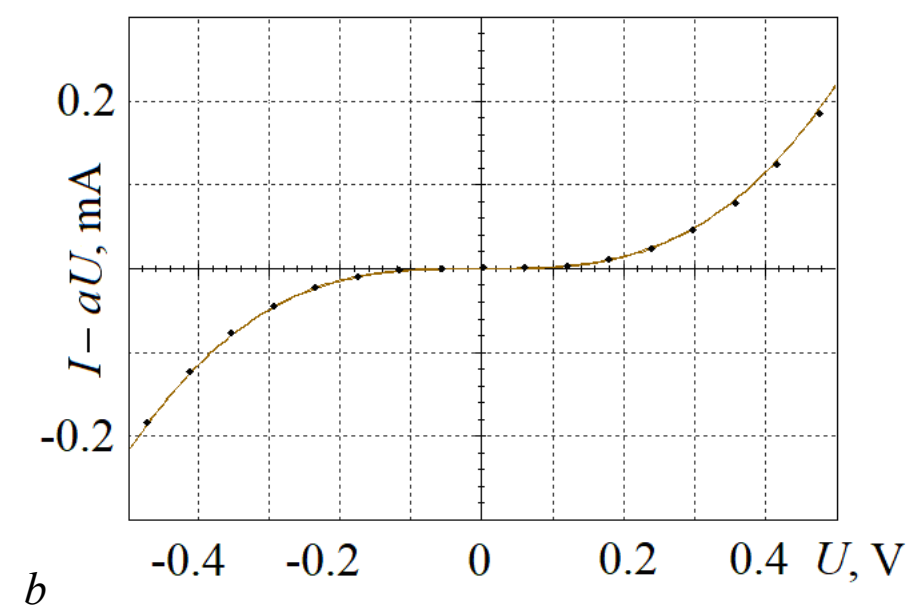

Рис. А1. Схема нелинейного элемента на полупроводниковых диодах (a) и нелинейная часть вольт-амперной характеристики для случая $R_{0}=706 \Omega, r_{0}=331 \Omega, r_{D}=2600 \Omega, \beta=60 \mathrm{~V}^{-2}(b)$. Сплошная линия - результат схемотехнического моделирования в среде Multisim, точки - расчет по формуле $I-a U=b U^{3}$, где $a$ и $b$ получены согласно (A4)

Fig. A1. Circuit diagram of nonlinear element on semiconductor diodes $(a)$ and nonlinear part of the current-voltage characteristic for the case of $R_{0}=706 \Omega, r_{0}=331 \Omega, r_{D}=2600 \Omega, \beta=60 \mathrm{~V}^{-2}(b)$. The solid line is the result of the circuit simulation in the Multisim environment, the dots are plotted using the formula $I-a U=b U^{3}$, where $a$ and $b$ are evaluated with (A4)

Приравнивая отдельно коэффициенты при первой и третьей степени $U$, получаем четыре уравнения относительно $a, b, c, d$. В итоге находим коэффициенты в разложении в ряд вольт-амперной характеристики составного элемента

$$
I=a U+b U^{3}
$$

в виде

$$
a=\frac{r_{0}^{-1}+r_{D}^{-1}}{1+R_{0}\left(r_{0}^{-1}+r_{D}^{-1}\right)}, \quad b=\frac{\beta r_{D}^{-1}}{\left[1+R_{0}\left(r_{0}^{-1}+r_{D}^{-1}\right)\right]^{4}} .
$$

На рис. A1, $b$ показана нелинейная часть вольт-амперной характеристики элемента с диодами $1 \mathrm{~N} 1200 \mathrm{C}$, в котором $R_{0}=706 \Omega, r_{0}=331 \Omega$. Из графика можно видеть хорошее соответствие характеристик, построенных по результатам моделирования в среде Multisim (сплошная линия) и по формуле (A3) с коэффициентами (A4).

\section{Библиографический список}

1. Dmitriev A.S., Panas A.I., Starkov S.O. Experiments on speech and music signals transmission using chaos // International Journal of Bifurcation and Chaos in Applied Sciences and Engineering. 1995. Vol. 5. P. 1249-1254.

2. Kennedy M., Setti G., Rovatti R. Chaotic electronics in telecommunications. CRC press, 2000.

3. Bollt E.M. Review of chaos communication by feedback control of symbolic dynamics // International Journal of Bifurcation and Chaos in Applied Sciences and Engineering. 2003. Vol. 13. P. 269-285.

4. Короновский А.А., Москаленко О.И., Храмов А.Е. О применении хаотической синхронизации для скрытой передачи информации // УФН. 2009. Т. 179. №12. С. 1281-1310.

5. Kaddoum G. Wireless chaos-based communication systems: A comprehensive survey // IEEE Access. 2016. Vol. 4. P. 2621-2648. 
6. Isaeva O.B., Jalnine A.Yu., Kuznetsov S.P. Chaotic communication with robust hyperbolic transmitter and receiver // IEEE Xplore. Progress In Electromagnetics Research Symposium. Proceedings: St Petersburg, Russia, 22-25 May 2017. P. 3129-3136.

7. Baptista M.S. Cryptography with chaos // Physics Letters A. 1998. Vol. 240, no. 1-2. P. 50-54.

8. Kocarev L. Chaos-based cryptography: a brief overview // IEEE Circuits and Systems Magazine. 2001. Vol. 1, no. 3. P. 6-21.

9. Dachselt F., Schwarz W. Chaos and cryptography // IEEE Transactions on Circuits and Systems I: Fundamental Theory and Applications. 2001. Vol. 48, no. 12. P. 1498-1509.

10. Antonik P., Gulina M., Pauwels J., Massar S. Using a reservoir computer to learn chaotic attractors, with applications to chaos synchronization and cryptography // Physical Review E. 2018. Vol. 98, no. 1. 012215.

11. Verschaffelt G., Khoder M., Van der Sande G. Random number generator based on an integrated laser with on-chip optical feedback // Chaos: An Interdisciplinary Journal of Nonlinear Science. 2017. Vol. 27. 114310.

12. Bakiri M., Guyeux C., Couchot J.F., Oudjida A.K. Survey on hardware implementation of random number generators on FPGA: Theory and experimental analyses // Computer Science Review. 2018. Vol. 27. P. 135-153.

13. Yeoh W.Z., Teh J.S., Chern H.R. A parallelizable chaos-based true random number generator based on mobile device cameras for the Android platform // Multimedia Tools and Applications. 2019. Vol. 78, no. 12. P. 15929-15949.

14. Karakaya B., Gülten A., Frasca M. A true random bit generator based on a memristive chaotic circuit: Analysis, design and FPGA implementation // Chaos, Solitons \& Fractals. 2019. Vol. 119. P. $143-149$.

15. Liu Z., Zhu X., Hu W., Jiang $F$. Principles of chaotic signal radar // International Journal of Bifurcation and Chaos in Applied Sciences and Engineering. 2007. Vol. 17. P. 1735-1739.

16. Pappu C.S., Flores B.C., Debroux P.S., Boehm J.E. An electronic implementation of Lorenz chaotic oscillator synchronization for bistatic radar applications // IEEE Transactions on Aerospace and Electronic Systems. 2017. Vol. 53, no. 4. P. 2001-2013.

17. Jiang T., Long J., Wang Z., Qiao S., Cui W., Ma W., Ran L. Experimental investigation of a direct chaotic signal radar with Colpitts oscillator // Journal of Electromagnetic Waves and Applications. 2010. Vol. 24, no. 8-9. P. 1229-1239.

18. Xiong G., Xi C., He J., Yu W. Radar target detection method based on cross-correlation singularity power spectrum // IET Radar, Sonar \& Navigation. 2019. Vol. 13, no. 5. P. 730-739.

19. Xu H., Li L., Li Y., Zhang J., Han H., Liu L., Li J. Chaos-based through-wall life-detection radar // International Journal of Bifurcation and Chaos in Applied Sciences and Engineering. 2019. Vol. 29. 1930020.

20. Banerjee S., Yorke J.A., Grebogi C. Robust chaos // Physical Review Letters. 1998. Vol. 80, no. 14. P. 3049-3052.

21. Potapov A., Ali M.K. Robust chaos in neural networks // Physics Letters A. 2000. Vol. 277, no. 6. P. 310-322.

22. Elhadj Z., Sprott J.C. On the robustness of chaos in dynamical systems: Theories and applications // Frontiers of Physics in China. 2008. Vol. 3, no. 2. P. 195-204.

23. Elhadj Z. and Sprott J.C. Robust Chaos and Its Applications. World Scientific, Singapore, 2011. $472 \mathrm{p}$. 
24. Glendinning P.A., Simpson D.J.W. Robust Chaos and the Continuity of Attractors // arXiv preprint arXiv: 1906.11974. 2019.

25. Gusso A., Dantas W. G., Ujevic S. Prediction of robust chaos in micro and nanoresonators under two-frequency excitation // Chaos: An Interdisciplinary Journal of Nonlinear Science. 2019. Vol. 29, no. 3. 033112.

26. Deshpande A., Chen Q., Wang Y., Lai Y.C., Do Y. Effect of smoothing on robust chaos // Physical Review E. 2010. Vol. 82, no. 2. 026209.

27. Shilnikov L. Mathematical problems of nonlinear dynamics: A tutorial // International Journal of Bifurcation and Chaos in Applied Sciences and Engineering. 1997. Vol. 7, no. 9. P. 1353-2001.

28. Gonchenko S.V., Shil'nikov L.P., Turaev D.V. Quasiattractors and homoclinic tangencies // Computers \& Mathematics with Applications. 1997. Vol. 34, no. 2-4. P. 195-227.

29. Botella-Soler V., Castelo J.M., Oteo J.A., Ros J. Bifurcations in the Lozi map // Journal of Physics A: Mathematical and Theoretical. 2011. Vol. 44, no. 30. 305101.

30. Elhadj Z. Lozi Mappings: Theory and Applications. CRC Press, 2013.

31. Belykh V.N., Belykh I. Belykh map // Scholarpedia. 2011. Vol. 6, no. 10. P. 5545.

32. Кузнецов С.П. Аттрактор Белых в отображении Заславского и его трансформация при сглаживании // Известия вузов. ПНД. 2018. Т. 26, № 1. С. 64-79.

33. Смейл С. Дифференцируемые динамические системы // Успехи математических наук. 1970. Т. 25, № 1 (151). С. 113-185.

34. Аносов Д.В., Арансон С.Х., Гринес В.З., Плыкин Р.В., Сатаев Е.А., Сафонов А.В., Солодов B.B., Старков А.Н., Степин А.М., Шлячков С.В. Динамические системы с гиперболическим поведением // Итоги науки и техники. Серия «Современные проблемы математики. Фундаментальные направления». М.: ВИНИТИ, 1991. Т. 66. 242 с.

35. Каток А.Б., Хасселблат Б. Введение в современную теорию динамических систем. М.: Факториал, 1999. 768c.

36. Hasselblatt B., Pesin Ya. Hyperbolic dynamics // Scholarpedia. 2008. Vol. 3, no. 6. C. 2208.

37. Pugh C., Peixoto M.M. Structural stability. Scholarpedia. 2008. Vol. 3, no. 9. C. 4008.

38. Аносов Д.В. Геодезические потоки на замкнутых римановых многообразиях отрицательной кривизны // Труды МИАН СССР. 1967. Т. 90. С. 3-210.

39. Аносов Д.В., Синай Я.Г. Некоторые гладкие эргодические системы // Успехи математических наук. 1967. Т. 22, № 5 (137). С. 107-172.

40. Синай Я. Г. Стохастичность динамических систем // Нелинейные волны. М.: Наука, 1979. C. $192-212$.

41. Kuznetsov S.P. Hyperbolic Chaos: A Physicist's View. Berlin, Heidelberg: Springer-Verlag, 2012. $336 \mathrm{p}$.

42. Кузнеиов С.П. Динамический хаос и гиперболические аттракторы: От математики к физике. Москва; Ижевск: ИКИ, 2013. 488 с.

43. Кузнецов С.П. Динамический хаос и однородно гиперболические аттракторы: От математики к физике // УФН. 2011. 181, № 2. С. 121-149.

44. Кузнеиов С.П., Селезнев Е.П. Хаотическая динамика в физической системе со странным аттрактором типа Смейла-Вильямса // ЖЭТФ. 2006. Т. 129, № 2. С. 400-412.

45. Кузнечов С.П., Пономаренко В.И. О возможности реализации странного аттрактора типа Смейла-Вильямса в радиотехническом генераторе с запаздыванием // Письма в ЖТФ. 2008. Т. 34, вып. 18. С. 1-8. 
46. Turukina L.V., Pikovsky A. Hyperbolic chaos in a system of resonantly coupled weakly nonlinear oscillators // Physics Letters A. 2011. Vol. 375, no. 11. P. 1407-1411.

47. Кузнецов С.П., Пономаренко В.И., Селезнев Е.П. Автономная система - генератор гиперболического хаоса. Схемотехническое моделирование и эксперимент // Известия вузов. ПНД. 2013. T. 21, № 5. C. 17-30.

48. Isaeva O.B., Kuznetsov S.P., Sataev I.R., Savin D.V., Seleznev E.P. Hyperbolic chaos and other phenomena of complex dynamics depending on parameters in a nonautonomous system of two alternately activated oscillators // International Journal of Bifurcation and Chaos in Applied Sciences and Engineering. 2015. Vol. 25, no. 12. 1530033.

49. Mangiarotti S., Letellier C. Topological analysis for designing a suspension of the Hénon map // Physics Letters A. 2015. Vol. 379, no. 47-48. P. 3069-3074.

50. Balazs N.L., Voros A. Chaos on the pseudosphere // Physics Reports. 1986. Vol. 143, no. 3. P. 109-240.

51. Hadamard $J$. Les surfaces à courbures opposées et leurs lignes géodésique // J. Math. Pures Appl. 1898. Vol. 4. P. 27-73.

52. Хернитер M.E. Multisim. Современная система компьютерного моделирования и анализа схем электронных устройств. М: Издательский дом «ДМК-пресс», 2006. 488 с.

53. Тёрстон У.П., Уикс Д.Р. Математика трехмерных многообразий // В мире науки. 1984, № 9. C. $74-88$.

54. Hunt T.J. and MacKay R.S. Anosov parameter values for the triple linkage and a physical system with a uniformly chaotic attractor // Nonlinearity. 2003. Vol. 16. P. 1499-1510.

55. Кузнецов С.П. Гиперболический хаос в автоколебательных системах на основе тройного шарнирного механизма: Проверка отсутствия касаний устойчивых и неустойчивых многообразий фазовых траекторий // Нелинейная динамика. 2016. Т.12. № 1. С. 121-143.

56. Meeks W.H., Pérez J. A Survey on Classical Minimal Surface Theory. University Lecture Series. Vol. 60. American Mathematical Society, 2012. 182 p.

57. Прасолов В.В. Наглядная топология. М.: МЦНМО, 1995. 111 с.

58. Гантмахер Ф.Р. Лекции по аналитической механике. 3-е изд. М.: Физматлит, 2005. 264 с.

59. Goldstein H., Poole Ch.P. Jr., Safko J.L. Classical Mechanics. 3d ed. Boston, Mass.: AddisonWesley, 2001. $680 \mathrm{p}$.

60. Лихарев К.К. Введение в динамику джозефсоновских переходов. М.: Наука, 1985. 320 с.

61. Шахгильдян B.B., Ляховкин А.А. Системы фазовой автоподстройки частоты. М.: Связь, 1972. $446 \mathrm{c}$.

62. Best R.E. Phase-Locked Loops: Design, Simulation and Applications. McGraw Hill, 2007. 490 p.

63. Kuznetsov S.P. From geodesic flow on a surface of negative curvature to electronic generator of robust chaos // International Journal of Bifurcation and Chaos in Applied Sciences and Engineering. 2016. Vol. 26, no. 14. 1650232.

64. Кузнеиов С.П. От динамики Аносова на поверхности отрицательной кривизны к электронному генератору грубого хаоса // Известия Саратовского университета - Новая серия. Серия Физика. 2016. Т. 16, № 3. С. 131-144.

65. Пиковский А.С., Рабинович М.И., Трахтенгери В.Ю. Возникновение стохастичности при распадном ограничении параметрической неустойчивости // ЖЭТФ. 1978. Т. 74. C. $1366-1374$.

66. Horowitz P. Build a Lorenz attractor: http://frank.harvard.edu/ paulh/misc/lorenz.htm 
67. Weady S., Agarwal S., Wilen L., Wettlaufer J.S. Circuit bounds on stochastic transport in the Lorenz equations // Physics Letters A. 2018. Vol. 382. P. 1731-1737.

68. Fitch A.L., Iu H.H., Lu D.D. An analog computer for electronic engineering education // IEEE Transactions on Education. 2010. Vol. 54, no. 4. P. 550-557.

69. Гонченко А.С., Гонченко С.В., Казаков А.О., Козлов А.Д. Математическая теория динамического хаоса и её приложения: Обзор. Часть 1. Псевдогиперболические аттракторы // Известия вузов. ПНД. 2017. Т. 25, № 2. С. 4-36.

70. Bonatti C., Diaz L.J., Viana M. Dynamics Beyond Uniform Hyperbolicity. A Global Geometric and Probobalistic Perspective. Berlin, Heidelberg, New-York: Springer, 2005. 384 p.

71. Свешников А.А. Прикладные методы теории случайных функций. М.: Наука, 1968. 464 с.

72. Дженкинс Г., Ваттс Д. Спектральный анализ и его приложения. Т. 2. М.: Мир, 1972. 287 с.

73. Benettin G., Galgani L., Giorgilli A., Strelcyn J.M. Lyapunov characteristic exponents for smooth dynamical systems and for Hamiltonian systems; a method for computing all of them // Meccanica. 1980. Vol. 15, no. 1. P. 9-20.

74. Shimada I., Nagashima T. A numerical approach to ergodic problem of dissipative dynamical systems // Progress of Theoretical Physics. 1979. Vol. 61, no. 6. P. 1605-1616.

75. Pikovsky A., Politi A. Lyapunov Exponents: A Tool to Explore Complex Dynamics. Cambridge University Press, 2016. 295 p.

76. Lai Y.-Ch., Grebogi C., Yorke J.A., Kan I. How often are chaotic saddles nonhyperbolic? // Nonlinearity. 1993. Vol. 6. P. 779-798.

77. Anishchenko V.S., Kopeikin A.S., Kurths J., Vadivasova T.E., Strelkova G.I. Studying hyperbolicity in chaotic systems // Physics Letters A. 2000. Vol. 270. P. 301-307.

78. Kuptsov P.V. Fast numerical test of hyperbolic chaos // Physical Review E. 2012. Vol. 85. 015203.

79. Круглов В.П. Методика и результаты численной проверки гиперболической природы аттракторов для редуцированных моделей распределенных систем // Известия вузов. ПНД. 2014. T. 22, № 6. C. 79-93.

80. Kuptsov P.V., Kuznetsov S.P. Numerical test for hyperbolicity of chaotic dynamics in time-delay systems // Physical Review E. 2016. Vol. 946 no. 1. 010201.

81. Kuptsov P.V., Kuznetsov S.P. Lyapunov analysis of strange pseudohyperbolic attractors: Angles between tangent subspaces, local volume expansion and contraction // Regular and Chaotic Dynamics. 2018. Vol. 23, no. 7-8. P. 908-932.

\section{References}

1. Dmitriev A.S., Panas A.I., Starkov S.O. Experiments on speech and music signals transmission using chaos. International Journal of Bifurcation and Chaos in Applied Sciences and Engineering, 1995, vol. 5, no. 4, pp. 1249-1254.

2. Kennedy M., Setti G., Rovatti R. Chaotic Electronics in Telecommunications. CRC press, 2000. $363 \mathrm{p}$.

3. Bollt E.M. Review of chaos communication by feedback control of symbolic dynamics. International Journal of Bifurcation and Chaos in Applied Sciences and Engineering, 2003, vol. 13, pp. 269-285.

4. Koronovskii A.A., Moskalenko O.I., Hramov A.E. On the use of chaotic synchronization for secure communication. Physics-Uspekhi, 2009, vol. 52, no. 12, pp. 1213-1238.

5. Kaddoum G. Wireless chaos-based communication systems: A comprehensive survey. IEEE Access, 2016, vol. 4, pp. 2621-2648. 
6. Isaeva O.B., Jalnine A.Yu., Kuznetsov S.P. Chaotic communication with robust hyperbolic transmitter and receiver. IEEE Xplore. Progress In Electromagnetics Research Symposium. Proceedings: St Petersburg, Russia, 22-25 May 2017, pp. 3129-3136.

7. Baptista M. S. Cryptography with chaos. Physics Letters A, 1998, vol. 240, no. 1-2, pp. 50-54.

8. Kocarev L. Chaos-based cryptography: A brief overview. IEEE Circuits and Systems Magazine, 2001, vol. 1, no. 3, pp. 6-21.

9. Dachselt F., Schwarz W. Chaos and cryptography. IEEE Transactions on Circuits and Systems I: Fundamental Theory and Applications, 2001, vol. 48, no. 12, pp. 1498-1509.

10. Antonik P., Gulina M., Pauwels J., Massar S. Using a reservoir computer to learn chaotic attractors, with applications to chaos synchronization and cryptography. Physical Review E, 2018, vol. 98 , no. $1,012215$.

11. Verschaffelt G., Khoder M., Van der Sande G. Random number generator based on an integrated laser with on-chip optical feedback. Chaos: An Interdisciplinary Journal of Nonlinear Science, 2017, vol. 27, 114310.

12. Bakiri M., Guyeux C., Couchot J.F., Oudjida A.K. Survey on hardware implementation of random number generators on FPGA: Theory and experimental analyses. Computer Science Review, 2018, vol. 27, pp. 135-153.

13. Yeoh W.Z., Teh J.S., Chern H.R. A parallelizable chaos-based true random number generator based on mobile device cameras for the Android platform. Multimedia Tools and Applications, 2019, vol. 78, no. 12, pp. 15929-15949.

14. Karakaya B., Gülten A., Frasca M. A true random bit generator based on a memristive chaotic circuit: Analysis, design and FPGA implementation. Chaos, Solitons \& Fractals, 2019, vol. 119, pp. 143-149.

15. Liu Z., Zhu X., Hu W., Jiang F. Principles of chaotic signal radar. International Journal of Bifurcation and Chaos in Applied Sciences and Engineering, 2007, vol. 17, pp. 1735-1739.

16. Pappu C.S., Flores B.C., Debroux P.S., Boehm J.E. An electronic implementation of Lorenz chaotic oscillator synchronization for bistatic radar applications. IEEE Transactions on Aerospace and Electronic Systems, 2017, vol. 53, no. 4, pp. 2001-2013.

17. Jiang T., Long J., Wang Z., Qiao S., Cui W., Ma W., Ran L. Experimental investigation of a direct chaotic signal radar with Colpitts oscillator. Journal of Electromagnetic Waves and Applications, 2010, vol. 24, no. 8-9, pp. 1229-1239.

18. Xiong G., Xi C., He J., Yu W. Radar target detection method based on cross-correlation singularity power spectrum. IET Radar, Sonar \& Navigation, 2019, vol. 13, no. 5, pp. 730-739.

19. Xu H., Li L., Li Y., Zhang J., Han H., Liu L., Li J. Chaos-based through-wall life-detection radar. International Journal of Bifurcation and Chaos in Applied Sciences and Engineering, 2019, vol. 29, 1930020.

20. Banerjee S., Yorke J.A., Grebogi C. Robust chaos. Physical Review Letters, 1998, vol. 80, no. 14, pp. 3049-3052.

21. Potapov A., Ali M.K. Robust chaos in neural networks. Physics Letters A, 2000, vol. 277, no. 6, pp. 310-322.

22. Elhadj Z., Sprott J.C. On the robustness of chaos in dynamical systems: Theories and applications. Frontiers of Physics in China, 2008, vol. 3, no. 2, pp. 195-204.

23. Elhadj Z. and Sprott J.C. Robust Chaos and Its Applications. Singapore: World Scientific, 2011. $472 \mathrm{p}$. 
24. Glendinning P.A., Simpson D.J.W. Robust Chaos and the Continuity of Attractors. arXiv preprint arXiv: 1906.11974, 2019.

25. Gusso A., Dantas W. G., Ujevic S. Prediction of robust chaos in micro and nanoresonators under two-frequency excitation. Chaos: An Interdisciplinary Journal of Nonlinear Science, 2019, vol. 29, no. 3, 033112.

26. Deshpande A., Chen Q., Wang Y., Lai Y. C., Do Y. Effect of smoothing on robust chaos. Physical Review E, 2010, vol. 82, no. 2, 026209.

27. Shilnikov L. Mathematical problems of nonlinear dynamics: A tutorial. International Journal of Bifurcation and Chaos in Applied Sciences and Engineering, 1997, vol. 7, no. 9, pp. 1353-2001.

28. Gonchenko S.V., Shil'nikov L.P., Turaev D.V. Quasiattractors and homoclinic tangencies. Computers \& Mathematics with Applications, 1997, vol. 34, no. 2-4, pp. 195-227.

29. Botella-Soler V., Castelo J.M., Oteo J.A., Ros J. Bifurcations in the Lozi map. Journal of Physics A: Mathematical and Theoretical, 2011, vol. 44, no. 30, 305101.

30. Elhadj Z. Lozi Mappings: Theory and Applications. CRC Press, 2013. 338 p.

31. Belykh V.N., Belykh I. Belykh map. Scholarpedia, 2011, vol. 6, no. 10, p. 5545.

32. Kuznetsov S.P. Belykh attractor in Zaslavsky map and its transformation under smoothing. Izvestiya VUZ. Applied Nonlinear Dynamics, 2018, vol. 26, no. 1, pp. $64-79$ (in Russian).

33. Smale S. Differentiable dynamical systems. Bull. Amer. Math. Soc. (NS), 1967, vol. 73, pp. 747-817.

34. Anosov D.V., Aranson S.Kh., Grines V.Z., Plykin R.V., Sataev E.A., Safonov A.V., Solodov V.V., Starkov A.N., Stepin A.M., Shlyachkov S.V. Dynamical Systems IX: Dynamical Systems with Hyperbolic Behaviour (Encyclopaedia of Mathematical Sciences, Vol. 9). Springer, 1995. 236 p.

35. Katok A. and Hasselblatt B. Introduction to the Modern Theory of Dynamical Systems, (Encyclopedia of Mathematics and its Applications, Vol. 54). Cambridge Univ. Press, 1996. 824 p.

36. Hasselblatt B., Pesin Ya. Hyperbolic dynamics. Scholarpedia, 2008, vol. 3, no. 6, p. 2208.

37. Pugh C., Peixoto M.M. Structural stability. Scholarpedia, 2008, vol. 3, no. 9, p. 4008.

38. Anosov D.V. Geodesic flows on closed Riemannian manifolds of negative curvature, Trudy Mat. Inst. Steklov, 1967, vol. 90, pp. 3-210 (in Russian).

39. Anosov D. V., Sinai Y. G. Some smooth ergodic systems. Russian Mathematical Surveys, 1967, vol. 22, no. 5, pp. 103-167.

40. Sinai Ya.G. The stochasticity of dynamical systems. Selected Translations. Selecta Math. Soviet., 1981, vol. 1, no. 1, pp. 100-119.

41. Kuznetsov S.P. Hyperbolic Chaos: A Physicist's View. Berlin, Heidelberg: Springer-Verlag, 2012. $336 \mathrm{p}$.

42. Kuznetsov S.P. Dynamical Chaos and Hyperbolic Attractors: From Mathematics to Physics. Moscow; Izhevsk: ICS, 2013, 488 p. (in Russian).

43. Kuznetsov S.P. Dynamical chaos and uniformly hyperbolic attractors: from mathematics to physics. Physics-Uspekhi, 2011, vol. 54, no. 2, pp. 119-144.

44. Kuznetsov S.P., Seleznev E.P. A strange attractor of the Smale-Williams type in the chaotic dynamics of a physical system. JETP, 2006, vol. 102, no. 2, pp. 355-364.

45. Kuznetsov S.P., Ponomarenko V.I. Realization of a strange attractor of the Smale-Williams type in a radiotechnical delay-feedback oscillator. Technical Physics Letters, 2008, vol. 34, no. 9, pp. $771-773$. 
46. Turukina L.V., Pikovsky A. Hyperbolic chaos in a system of resonantly coupled weakly nonlinear oscillators. Physics Letters A, 2011, vol. 375, no. 11, pp. 1407-1411.

47. Ponomarenko V.I., Seleznev E.P., Kuznetsov S.P. Autonomous system generating hyperbolic chaos: Circuit simulation and experiment. Izvestiya VUZ, Applied Nonlinear Dynamics, 2013, vol. 21, no. 5, pp. 17-30 (in Russian).

48. Isaeva O.B., Kuznetsov S.P., Sataev I.R., Savin D.V., Seleznev E.P. Hyperbolic chaos and other phenomena of complex dynamics depending on parameters in a nonautonomous system of two alternately activated oscillators. International Journal of Bifurcation and Chaos in Applied Sciences and Engineering, 2015, vol. 25, no. 12, 1530033.

49. Mangiarotti S., Letellier C. Topological analysis for designing a suspension of the Hénon map. Physics Letters A, 2015, vol. 379, no. 47-48, pp. 3069-3074.

50. Balazs N.L., Voros A. Chaos on the pseudosphere. Physics Reports, 1986, vol. 143, no. 3, pp. 109-240.

51. Hadamard J. Les surfaces à courbures opposées et leurs lignes géodésique. J. Math. Pures Appl., 1898, vol. 4, pp. 27-73.

52. Herniter M.E. Schematic Capture with Multisim. Prentice Hall, 2004.

53. Thurston W.P. and Weeks J.R., The mathematics of three-dimensional manifolds, Scientific American, 1984, vol. 251, pp. 94-106.

54. Hunt T.J., MacKay R.S. Anosov parameter values for the triple linkage and a physical system with a uniformly chaotic attractor. Nonlinearity, 2003, vol. 16, pp. 1499-1510.

55. Kuznetsov S.P. Hyperbolic chaos in self-oscillating systems based on mechanical triple linkage: Testing absence of tangencies of stable and unstable manifolds for phase trajectories. Regular and Chaotic Dynamics, 2015, vol. 20, no. 6, pp. 649-666.

56. Meeks W. H., Pérez J., Pérez J. A Survey on Classical Minimal Surface Theory. University Lecture Series, vol.60. American Mathematical Society, 2012, 182 p.

57. Prasolov V.V. Intuitive topology. American Mathematical Society, 1995, 93 p.

58. Gantmacher F.R. Lectures in Analytical Mechanics, Moscow: Mir, 1975, 264 p.

59. Goldstein, H., Poole, Ch.P. Jr., Safko, J. L., Classical Mechanics, 3d ed., Boston, Mass.: AddisonWesley, 2001. $680 \mathrm{p}$.

60. Likharev K.K. Dynamics of Josephson Junctions and Circuits. CRC Press, 1986. 614 p.

61. Shakhgildyan V.V. and Lyahovkin A.A. Phase-Locked Loops. Moscow, Svyaz', 1972. 446 p. (in Russian).

62. Best R.E. Phase-Locked Loops: Design, Simulation and Applications. McGraw Hill, 2007. 490 p.

63. Kuznetsov S.P. From geodesic flow on a surface of negative curvature to electronic generator of robust chaos. International Journal of Bifurcation and Chaos in Applied Sciences and Engineering, 2016, vol. 26, no. 14. 1650232.

64. Kuznetsov S.P. From Anosov's dynamics on a surface of negative curvature to electronic generator of robust chaos. Izvestiya of Saratov University. New series. Series Physics, 2016, vol. 16, iss. 3 , pp. 131-144.

65. Pikovski A.S., Rabinovich M.I., Trakhtengerts V.Y. Appearance of chaos at decay saturation of parametric instability. Sov. Phys. JETP, 1978, vol. 47, pp. 715-719.

66. Horowitz P. Build a Lorenz attractor: http://frank.harvard.edu/ paulh/misc/lorenz.htm

67. Weady S., Agarwal S., Wilen L., Wettlaufer J.S. Circuit bounds on stochastic transport in the Lorenz equations. Physics Letters A, 2018, vol. 382, pp. 1731-1737.

68. Fitch A.L., Iu H.H., Lu D.D. An analog computer for electronic engineering education. IEEE Transactions on Education, 2010, vol. 54, no. 4, pp. 550-557. 
69. Gonchenko A.S., Gonchenko S.V., Kazakov A.O., Kozlov A.D. Mathematical theory of dynamical chaos and its applications: Review. Part 1. Pseudohyperbolic attractors. Izvestiya VUZ. Applied Nonlinear Dynamics, 2017, vol. 25, no. 2, pp. 4-36 (in Russian).

70. Bonatti C., Diaz L.J., Viana M. Dynamics Beyond Uniform Hyperbolicity. A Global Geometric and Probobalistic Perspective. Encyclopedia of Mathematical Sciences, vol.102. Berlin, Heidelberg, New-York: Springer, 2005. 384 p.

71. Sveshnikov A.A. Applied Methods of the Theory of Random Functions. Elsevier, 2014. 332 p.

72. Jenkins G.M., Watts D.G. Spectral Aalysis and its Applications. Holden-Day, 1969. 525 p.

73. Benettin G., Galgani L., Giorgilli A., Strelcyn J.M. Lyapunov characteristic exponents for smooth dynamical systems and for Hamiltonian systems; a method for computing all of them. Meccanica, 1980, vol. 15, no. 1, pp. 9-20.

74. Shimada I., Nagashima T. A numerical approach to ergodic problem of dissipative dynamical systems. Progress of Theoretical Physics, 1979, vol. 61, no. 6, pp. 1605-1616.

75. Pikovsky A., Politi A. Lyapunov Exponents: A Tool to Explore Complex Dynamics. Cambridge University Press, 2016. 295 p.

76. Lai Y.-Ch., Grebogi C., Yorke J.A., and Kan I. How often are chaotic saddles nonhyperbolic? Nonlinearity, 1993, vol. 6, pp. 779-798.

77. Anishchenko V.S., Kopeikin A.S., Kurths J., Vadivasova T.E., Strelkova G.I. Studying hyperbolicity in chaotic systems. Physics Letters A, 2000, vol. 270, pp. 301-307.

78. Kuptsov P.V. Fast numerical test of hyperbolic chaos. Physical Review E, 2012, vol. 85, 015203.

79. Kruglov V. P. Technique and results of numerical test for hyperbolic nature of attractors for reduced models of distributed systems. Izvestiya VUZ. Applied Nonlinear Dynamics, 2014, vol. 22, iss. 6, pp. 79-93 (in Russian).

80. Kuptsov P.V., Kuznetsov S.P. Numerical test for hyperbolicity of chaotic dynamics in time-delay systems. Physical Review E, 2016, vol. 94, no. 1, 010201.

81. Kuptsov P.V., Kuznetsov S.P. Lyapunov analysis of strange pseudohyperbolic attractors: Angles between tangent subspaces, local volume expansion and contraction. Regular and Chaotic Dynamics, 2018, vol. 23, no. 7-8, pp. 908-932.

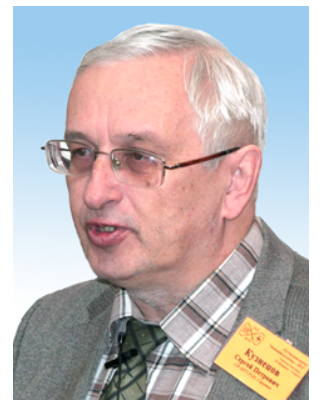

Кузнечов Сергей Петрович - родился в Москве (1951). Окончил Саратовский государственный университет (1973). Защитил диссертацию на соискание ученой степени кандидата физико-математических наук (1977) и доктора наук (1987) по специальности радиофизика в СГУ. Главный научный сотрудник Саратовского филиала Института радиотехники и электроники им. В.А. Котельникова РАН, где работает с 1988 г. и возглавляет лабораторию теоретической нелинейной динамики. Главный научный сотрудник лаборатории нелинейного анализа и конструирования новых средств передвижения Удмуртского государственного университета. Автор монографий «Strange Nonchaotic Attractors» (совместно с А. Пиковским и У. Фойдель), «Динамический хаос и гиперболические аттракторы: От математики к физике» и «Hyperbolic Chaos: a Physicist's View». Автор учебно-научных монографий «Нелинейные колебания» (в соавторстве с А.П. Кузнецовым и Н.М. Рыскиным) и «Динамический хаос». Опубликовал свыше 200 научных статей по нелинейной динамике, радиофизике и электронике. Под руководством С.П. Кузнецова защищены двенадцать кандидатских диссертаций. Лауреат государственной научной стипендии для ученых России (1994-1996). Член редакционной коллегии журналов «Известия вузов. Прикладная нелинейная динамика», «Regular and Chaotic Dynamics», «Russian Journal of Nonlinear Dynamics», «Известия Саратовского университета - Новая серия. Серия Физика».

Россия, 410019 Саратов, ул. Зеленая, 38

Институт радиотехники и электроники им. В.А. Котельникова РАН, Саратовский филиал E-mail: spkuz@yandex.ru 